\title{
Analysis of the scalar, axialvector, vector, tensor doubly charmed tetraquark states with QCD sum rules
}

\author{
Zhi-Gang Wang ${ }^{\mathrm{a}}$, Ze-Hui Yan \\ Department of Physics, North China Electric Power University, Baoding 071003, People's Republic of China
}

Received: 31 October 2017 / Accepted: 22 December 2017 / Published online: 12 January 2018

(C) The Author(s) 2018. This article is an open access publication

\begin{abstract}
In this article, we construct the axialvectordiquark-axialvector-antidiquark type currents to interpolate the scalar, axialvector, vector, tensor doubly charmed tetraquark states, and study them with QCD sum rules systematically by carrying out the operator product expansion up to the vacuum condensates of dimension 10 in a consistent way, the predicted masses can be confronted with the experimental data in the future. We can search for those doubly charmed tetraquark states in the Okubo-Zweig-Iizuka super-allowed strong decays to the charmed-meson pairs.
\end{abstract}

\section{Introduction}

Recently, the LHCb collaboration observed the doubly charmed baryon $\Xi_{c c}^{++}$in the $\Lambda_{c}^{+} K^{-} \pi^{+} \pi^{+}$mass spectrum, and obtained the mass $M_{\Xi_{c c}^{++}}=3621.40 \pm 0.72 \pm 0.27 \pm$ $0.14 \mathrm{MeV}$, but did not measure the spin [1]. The doubly heavy baryon configuration $Q Q q$ is very similar to the heavylight meson $\bar{Q} q$, where we have a doubly heavy diquark $Q Q$ instead of a heavy antiquark $\bar{Q}$ in color antitriplet. The attractive interaction induced by one-gluon exchange favors formation of the diquarks in color antitriplet $[2,3]$, the favored configurations are the scalar $\left(C \gamma_{5}\right)$ and axialvector $\left(C \gamma_{\mu}\right)$ diquark states [4-6]. For the $c c$ quark system, only the axialvector diquark $\varepsilon^{i j k} c_{j}^{T} C \gamma_{\mu} c_{k}$ and tensor diquark $\varepsilon^{i j k} c_{j}^{T} C \sigma_{\mu \nu} c_{k}$ survive due to the Fermi-Dirac statistics, the axialvector diquark $\varepsilon^{i j k} c_{j}^{T} C \gamma_{\mu} c_{k}$ is more stable than the tensor diquark $\varepsilon^{i j k} c_{j}^{T} C \sigma_{\mu \nu} c_{k}$, the observation of $\Xi_{c c}^{++}$indicates that there exists a strong correlation between the two charm quarks. We can take the diquark $\varepsilon^{i j k} c_{i}^{T} C \gamma_{\mu} c_{j}$ as basic constituent to construct the spin $\frac{1}{2}$ current

$J_{\Xi_{c c}}(x)=\varepsilon^{i j k} c_{i}^{T}(x) C \gamma_{\mu} c_{j}(x) \gamma_{5} \gamma^{\mu} u_{k}(x)$,

a e-mail: zgwang@aliyun.com or the spin $\frac{3}{2}$ current

$J_{\Xi_{c c}}^{\mu}(x)=\varepsilon^{i j k} c_{i}^{T}(x) C \gamma^{\mu} c_{j}(x) u_{k}(x)$,

to study $\Xi_{c c}^{++}$with the QCD sum rules [7-13].

The doubly heavy tetraquark state $Q Q \bar{q} \bar{q}^{\prime}$ is very similar to the doubly heavy baryon state $Q Q q$, where we have a light antidiquark $\bar{q} \bar{q}^{\prime}$ instead of a light quark $q$ in color triplet. The observation of $\Xi_{c c}^{++}$provides the crucial experimental input on the strong correlation between the two charm quarks, which may shed light on the spectroscopy of the doubly charmed tetraquark states. An axialvector doubly charmed diquark state can combine with an axialvector or scalar light antidiquark state to form a compact doubly charmed tetraquark state, it is interesting to revisit this subject with the QCD sum rules. The QCD sum rules approach is a powerful theoretical tool in studying the ground state hadrons, and it has given many successful descriptions of the hadronic parameters on the phenomenological side [1416]. Up to now, no experimental candidates for the doubly charmed tetraquark states $c c \bar{q} \bar{q}^{\prime}$ or $q q^{\prime} \bar{c} \bar{c}$ have been observed. There have been several works on the doubly heavy tetraquark states, such as potential quark models [17-23], QCD sum rules [24-26], heavy quark symmetry [27-29], lattice QCD [30-32], etc.

In previous work, we study the axialvector doubly heavy tetraquark states, which consist of an axialvector diquark ${ }^{i j k} Q_{j}^{T} C \gamma_{\mu} Q_{k}$ and a scalar antidiquark $\varepsilon^{i j k} \bar{q}_{j}^{T} \gamma_{5} C \bar{q}_{k}^{\prime}$, with the QCD sum rules in detail by taking into account the energy-scale dependence of the QCD spectral densities [33]. In this article, we choose the axialvector diquark ${ }^{i j k} c_{j}^{T} C \gamma_{\mu} c_{k}$ and axialvector antidiquark $\varepsilon^{i j k} \bar{q}_{j}^{T} \gamma_{\mu} C \bar{q}_{k}^{\prime}$ to construct the currents to interpolate the doubly charmed tetraquark states with the spin-parity $J^{P}=0^{+}, 1^{ \pm}, 2^{+}$, and study them with the QCD sum rules systematically by taking into account the contributions of the vacuum condensates up to dimension 10 in a consistent way in the operator product expansion. 
The article is arranged as follows: we derive the QCD sum rules for the masses and pole residues of the doubly charmed tetraquark states in Sect. 2; in Sect. 3, we present the numerical results and discussions; and Sect. 4 is reserved for our conclusion.

\section{The QCD sum rules for the doubly charmed tetraquark states}

In the following, we write down the two-point correlation functions $\Pi_{0}(p), \Pi_{\mu \nu \alpha \beta ; 1}(p)$ and $\Pi_{\mu \nu \alpha \beta ; 2}(p)$ in the QCD sum rules,

$$
\begin{gathered}
\Pi_{0}(p)=i \int d^{4} x e^{i p \cdot x}\left\langle 0\left|T\left\{J_{0}(x) J_{0}^{\dagger}(0)\right\}\right| 0\right\rangle, \\
\Pi_{\mu \nu \alpha \beta ; 1}(p)=i \int d^{4} x e^{i p \cdot x}\left\langle 0\left|T\left\{J_{\mu \nu ; 1}(x) J_{\alpha \beta ; 1}^{\dagger}(0)\right\}\right| 0\right\rangle, \\
\Pi_{\mu \nu \alpha \beta ; 2}(p)=i \int d^{4} x e^{i p \cdot x}\left\langle 0\left|T\left\{J_{\mu \nu ; 2}(x) J_{\alpha \beta ; 2}^{\dagger}(0)\right\}\right| 0\right\rangle,
\end{gathered}
$$

where $J_{0}(x)=J_{\bar{u} \bar{d} ; 0}(x), J_{\bar{u} \bar{s} ; 0}(x), J_{\bar{s} \bar{s} ; 0}(x), J_{\mu \nu ; 1}(x)=$ $J_{\mu v ; \bar{u} \bar{d} ; 1}(x), J_{\mu v ; \bar{u} ; ; 1}(x), J_{\mu v ; \bar{s} \bar{s} ; 1}(x), J_{\mu \nu ; 2}(x)=J_{\mu \nu ; \bar{u} \bar{d} ; 2}$ $(x), J_{\mu v ; \bar{u} ; 2}(x), J_{\mu v ; \bar{s} ; 2}(x)$,

$$
\begin{aligned}
J_{\bar{u} \bar{j} ; 0}(x)= & \varepsilon^{i j k} \varepsilon^{i m n} c_{i}^{T}(x) C \gamma_{\mu} c_{j}(x) \bar{u}_{m}(x) \gamma^{\mu} C \bar{d}_{n}^{T}(x), \\
J_{\bar{u} \bar{s} ; 0}(x)= & \varepsilon^{i j k} \varepsilon^{i m n} c_{i}^{T}(x) C \gamma_{\mu} c_{j}(x) \bar{u}_{m}(x) \gamma^{\mu} C \bar{s}_{n}^{T}(x), \\
J_{\bar{s} \bar{s} ; 0}(x)= & \varepsilon^{i j k} \varepsilon^{i m n} c_{i}^{T}(x) C \gamma_{\mu} c_{j}(x) \bar{s}_{m}(x) \gamma^{\mu} C \bar{s}_{n}^{T}(x), \\
J_{\mu \nu ; \bar{u} \bar{j} ; 1}(x)= & \varepsilon^{i j k} \varepsilon^{i m n}\left[c_{i}^{T}(x) C \gamma_{\mu} c_{j}(x) \bar{u}_{m}(x) \gamma_{\nu} C \bar{d}_{n}^{T}(x)\right. \\
& \left.-c_{i}^{T}(x) C \gamma_{\nu} c_{j}(x) \bar{u}_{m}(x) \gamma_{\mu} C \bar{d}_{n}^{T}(x)\right], \\
J_{\mu \nu ; \bar{u} \bar{s} ; 1}(x)= & \varepsilon^{i j k} \varepsilon^{i m n}\left[c_{i}^{T}(x) C \gamma_{\mu} c_{j}(x) \bar{u}_{m}(x) \gamma_{\nu} C \bar{s}_{n}^{T}(x)\right. \\
& \left.-c_{i}^{T}(x) C \gamma_{\nu} c_{j}(x) \bar{u}_{m}(x) \gamma_{\mu} C \bar{s}_{n}^{T}(x)\right], \\
J_{\mu v ; \bar{s} ; 1}(x)= & \varepsilon^{i j k} \varepsilon^{i m n}\left[c_{i}^{T}(x) C \gamma_{\mu} c_{j}(x) \bar{s}_{m}(x) \gamma_{\nu} C \bar{s}_{n}^{T}(x)\right. \\
& \left.-c_{i}^{T}(x) C \gamma_{\nu} c_{j}(x) \bar{s}_{m}(x) \gamma_{\mu} C \bar{s}_{n}^{T}(x)\right], \\
J_{\mu \nu ; \bar{u} \bar{j} ; 2}(x)= & \varepsilon^{i j k} \varepsilon^{i m n}\left[c_{i}^{T}(x) C \gamma_{\mu} c_{j}(x) \bar{u}_{m}(x) \gamma_{\nu} C \bar{d}_{n}^{T}(x)\right. \\
& \left.+c_{i}^{T}(x) C \gamma_{\nu} c_{j}(x) \bar{u}_{m}(x) \gamma_{\mu} C \bar{d}_{n}^{T}(x)\right], \\
J_{\mu \nu ; \bar{u} \bar{s} ; 2}(x)= & \varepsilon^{i j k} \varepsilon^{i m n}\left[c_{i}^{T}(x) C \gamma_{\mu} c_{j}(x) \bar{u}_{m}(x) \gamma_{\nu} C \bar{s}_{n}^{T}(x)\right. \\
& \left.+c_{i}^{T}(x) C \gamma_{\nu} c_{j}(x) \bar{u}_{m}(x) \gamma_{\mu} C \bar{s}_{n}^{T}(x)\right],
\end{aligned}
$$

$$
\begin{aligned}
J_{\mu \nu ; \bar{s} ; 2}(x)= & \varepsilon^{i j k} \varepsilon^{i m n}\left[c_{i}^{T}(x) C \gamma_{\mu} c_{j}(x) \bar{s}_{m}(x) \gamma_{\nu} C \bar{s}_{n}^{T}(x)\right. \\
& \left.+c_{i}^{T}(x) C \gamma_{\nu} c_{j}(x) \bar{s}_{m}(x) \gamma_{\mu} C \bar{s}_{n}^{T}(x)\right]
\end{aligned}
$$

the $i, j, k, m, n$ are color indices, $C$ is the charge conjugation matrix. We choose the currents $J_{0}(x), J_{\mu \nu ; 1}(x)$ and $J_{\mu \nu ; 2}(x)$ to interpolate the spin-parity $J^{P}=0^{+}, 1^{ \pm}$and $2^{+}$doubly charmed tetraquark states, respectively.

On the phenomenological side, we insert a complete set of intermediate hadronic states with the same quantum numbers as the current operators $J_{0}(x), J_{\mu \nu ; 1}(x)$ and $J_{\mu \nu ; 2}(x)$ into the correlation functions $\Pi_{0}(p), \Pi_{\mu \nu \alpha \beta ; 1}(p)$ and $\Pi_{\mu \nu \alpha \beta ; 2}(p)$ respectively to obtain the hadronic representation [14-16], and isolate the ground state contributions,

$$
\begin{aligned}
\Pi_{0}(p)= & \frac{\lambda_{Z}^{2}}{M_{Z}^{2}-p^{2}}+\cdots \\
= & \Pi_{0}\left(p^{2}\right) \\
\Pi_{\mu \nu \alpha \beta ; 1}(p)= & \frac{\lambda_{Z}^{2}}{M_{Z}^{2}-p^{2}}\left(p^{2} g_{\mu \alpha} g_{\nu \beta}-p^{2} g_{\mu \beta} g_{\nu \alpha}-g_{\mu \alpha} p_{\nu} p_{\beta}\right. \\
& \left.-g_{\nu \beta} p_{\mu} p_{\alpha}+g_{\mu \beta} p_{\nu} p_{\alpha}+g_{\nu \alpha} p_{\mu} p_{\beta}\right) \\
& +\frac{\lambda_{Y}^{2}}{M_{Y}^{2}-p^{2}}\left(-g_{\mu \alpha} p_{\nu} p_{\beta}-g_{\nu \beta} p_{\mu} p_{\alpha}\right. \\
& \left.+g_{\mu \beta} p_{\nu} p_{\alpha}+g_{\nu \alpha} p_{\mu} p_{\beta}\right)+\cdots \\
= & \Pi_{Z}\left(p^{2}\right)\left(p^{2} g_{\mu \alpha} g_{\nu \beta}-p^{2} g_{\mu \beta} g_{\nu \alpha}\right. \\
& -g_{\mu \alpha} p_{\nu} p_{\beta}-g_{\nu \beta} p_{\mu} p_{\alpha}+g_{\mu \beta} p_{\nu} p_{\alpha} \\
& \left.+g_{\nu \alpha} p_{\mu} p_{\beta}\right)+\Pi_{Y}\left(p^{2}\right)\left(-g_{\mu \alpha} p_{\nu} p_{\beta}\right. \\
& \left.-g_{\nu \beta} p_{\mu} p_{\alpha}+g_{\mu \beta} p_{\nu} p_{\alpha}+g_{\nu \alpha} p_{\mu} p_{\beta}\right) \\
\Pi_{\mu \nu \alpha \beta ; 2}(p)= & \frac{\lambda_{Z}^{2}}{M_{Z}^{2}-p^{2}}\left(\frac{\widetilde{g}_{\mu \alpha} \tilde{g}_{\nu \beta}+\widetilde{g}_{\mu \beta} \tilde{g}_{\nu \alpha}}{2}\right. \\
& \left.-\frac{\widetilde{g}_{\mu \nu} \tilde{g}_{\alpha \beta}}{3}\right)+\cdots, \\
= & \Pi_{2}\left(p^{2}\right)\left(\frac{\widetilde{g}_{\mu \alpha} \widetilde{g}_{\nu \beta}+\widetilde{g}_{\mu \beta} \widetilde{g}_{\nu \alpha}}{2}-\frac{\widetilde{g}_{\mu \nu} \tilde{g}_{\alpha \beta}}{3}\right)
\end{aligned}
$$

where $\tilde{g}_{\mu \nu}=g_{\mu \nu}-\frac{p_{\mu} p_{v}}{p^{2}}$, the pole residues $\lambda_{Z}$ and $\lambda_{Y}$ are defined by

$$
\begin{aligned}
\left\langle 0\left|J_{0}(0)\right| Z_{0^{+}}(p)\right\rangle & =\lambda_{Z}, \\
\left\langle 0\left|J_{\mu \nu ; 1}(0)\right| Z_{1^{+}}(p)\right\rangle & =\frac{\lambda_{Z}}{M_{Z}} \epsilon_{\mu \nu \alpha \beta} \varepsilon^{\alpha} p^{\beta}, \\
\left\langle 0\left|J_{\mu \nu ; 1}(0)\right| Y_{1^{-}}(p)\right\rangle & =\frac{\lambda_{Y}}{M_{Y}}\left(\varepsilon_{\mu} p_{\nu}-\varepsilon_{v} p_{\mu}\right), \\
\left\langle 0\left|J_{\mu \nu ; 2}(0)\right| Z_{2^{+}}(p)\right\rangle & =\lambda_{Z} \varepsilon_{\mu \nu},
\end{aligned}
$$


$\varepsilon_{\mu}$ and $\varepsilon_{\mu \nu}$ are the polarization vectors of the spin $J=1$ and 2 tetraquark states, respectively. The summation of the polarization vectors $\varepsilon_{\mu}$ and $\varepsilon_{\mu \nu}$ results in the following formula:

$$
\begin{aligned}
\sum_{\lambda} \varepsilon_{\mu}^{*}(\lambda, p) \varepsilon_{\nu}(\lambda, p) & =-g_{\mu \nu}+\frac{p_{\mu} p_{v}}{p^{2}} \\
\sum_{\lambda} \varepsilon_{\alpha \beta}^{*}(\lambda, p) \varepsilon_{\mu \nu}(\lambda, p) & =\frac{\widetilde{g}_{\alpha \mu} \widetilde{g}_{\beta \nu}+\widetilde{g}_{\alpha \nu} \widetilde{g}_{\beta \mu}}{2}-\frac{\widetilde{g}_{\alpha \beta} \widetilde{g}_{\mu \nu}}{3} .
\end{aligned}
$$

The components $\Pi_{0}\left(p^{2}\right), \Pi_{Z}\left(p^{2}\right), \Pi_{Y}\left(p^{2}\right)$ and $\Pi_{2}\left(p^{2}\right)$ receive contributions of the hadronic states with the spinparity $J^{P}=0^{+}, 1^{+}, 1^{-}$and $2^{+}$, respectively.

Now we project out the components $\Pi_{Z}\left(p^{2}\right)$ and $\Pi_{Y}\left(p^{2}\right)$ by introducing the operators $P_{Z}^{\mu \nu \alpha \beta}$ and $P_{Y}^{\mu \nu \alpha \beta}$,

$$
\begin{aligned}
& \Pi_{1 ; A}\left(p^{2}\right)=p^{2} \Pi_{Z}\left(p^{2}\right)=P_{Z}^{\mu \nu \alpha \beta} \Pi_{\mu \nu \alpha \beta ; 1}(p), \\
& \Pi_{1 ; V}\left(p^{2}\right)=p^{2} \Pi_{Y}\left(p^{2}\right)=P_{Y}^{\mu \nu \alpha \beta} \Pi_{\mu \nu \alpha \beta ; 1}(p),
\end{aligned}
$$

where

$$
\begin{aligned}
& P_{Z}^{\mu \nu \alpha \beta}=\frac{1}{6}\left(g^{\mu \alpha}-\frac{p^{\mu} p^{\alpha}}{p^{2}}\right)\left(g^{\nu \beta}-\frac{p^{\nu} p^{\beta}}{p^{2}}\right), \\
& P_{Y}^{\mu \nu \alpha \beta}=\frac{1}{6}\left(g^{\mu \alpha}-\frac{p^{\mu} p^{\alpha}}{p^{2}}\right)\left(g^{\nu \beta}-\frac{p^{\nu} p^{\beta}}{p^{2}}\right)-\frac{1}{6} g^{\mu \alpha} g^{\nu \beta} .
\end{aligned}
$$

In this article, we carry out the operator product expansion for the correlation functions $\Pi_{0}(p), \Pi_{\mu \nu \alpha \beta ; 1}(p)$ and $\Pi_{\mu \nu \alpha \beta ; 2}(p)$ to the vacuum condensates up to dimension-10, and take into account the vacuum condensates which are vacuum expectations of the operators of the orders $\mathcal{O}\left(\alpha_{s}^{k}\right)$ with $k \leq 1$ in a consistent way [34-39], then we project out the components

$$
\begin{aligned}
& \Pi_{1 ; A}\left(p^{2}\right)=P_{Z}^{\mu \nu \alpha \beta} \Pi_{\mu \nu \alpha \beta ; 1}(p), \\
& \Pi_{1 ; V}\left(p^{2}\right)=P_{Y}^{\mu \nu \alpha \beta} \Pi_{\mu \nu \alpha \beta ; 1}(p),
\end{aligned}
$$

on the QCD side, and obtain the QCD spectral densities through dispersion relation,

$$
\begin{aligned}
\rho_{0}(s) & =\frac{\operatorname{Im} \Pi_{0}(s)}{\pi}, \\
\rho_{1 ; A}(s) & =\frac{\operatorname{Im} \Pi_{1 ; A}(s)}{\pi}, \\
\rho_{1 ; V}(s) & =\frac{\operatorname{Im} \Pi_{1 ; V}(s)}{\pi}, \\
\rho_{2}(s) & =\frac{\operatorname{Im} \Pi_{2}(s)}{\pi},
\end{aligned}
$$

where $\rho_{0}(s)=\rho_{\bar{u} \bar{d} ; 0}(s), \rho_{\bar{u} \bar{s} ; 0}(s), \rho_{\bar{s} \bar{s} ; 0}(s), \rho_{1 ; A}(s)=$ $\rho_{\bar{u} \bar{d} ; 1 ; A}(s), \rho_{\bar{u} \bar{s} ; 1 ; A}(s), \rho_{\bar{s} \bar{s} ; 1 ; A}(s), \rho_{1 ; V}(s)=\rho_{\bar{u} \bar{d} ; 1 ; V}(s)$, $\rho_{\bar{u} \bar{s} ; 1 ; V}(s), \rho_{\bar{s} \bar{s} ; 1 ; V}(s), \rho_{2}(s)=\rho_{\bar{u} \bar{d} ; 2}(s), \rho_{\bar{u} \bar{s} ; 2}(s), \rho_{\bar{s} \bar{s} ; 2}(s)$. The explicit expressions of the QCD spectral densities are given in the "Appendix".
Once the analytical expressions of the QCD spectral densities $\rho_{0}(s), \rho_{1 ; A}(s), \rho_{1 ; V}(s), \rho_{2}(s)$ are obtained, we can take the quark-hadron duality below the continuum thresholds $s_{0}$ and perform Borel transform with respect to the variable $P^{2}=-p^{2}$ to obtain the QCD sum rules,

$\lambda_{Z / Y}^{2} \exp \left(-\frac{M_{Z / Y}^{2}}{T^{2}}\right)=\int_{4 m_{c}^{2}}^{s_{0}} d s \rho(s) \exp \left(-\frac{s}{T^{2}}\right)$,

where $\rho(s)=\rho_{0}(s), \rho_{1 ; A}(s), \rho_{1 ; V}(s), \rho_{2}(s)$.

We derive Eq. (14) with respect to $\tau=\frac{1}{T^{2}}$, then eliminate the pole residues $\lambda_{Z / Y}$ to obtain the QCD sum rules for the masses of the doubly charmed tetraquark states,

$M_{Z / Y}^{2}=\frac{-\frac{\mathrm{d}}{\mathrm{d} \tau} \int_{4 m_{c}^{2}}^{s_{0}} \mathrm{~d} s \rho(s) \mathrm{e}^{-\tau s}}{\int_{4 m_{c}^{2}}^{s_{0}} \mathrm{~d} s \rho(s) \mathrm{e}^{-\tau s}}$

\section{Numerical results and discussions}

We take the standard values of the vacuum condensates $\langle\bar{q} q\rangle=-(0.24 \pm 0.01 \mathrm{GeV})^{3},\left\langle\bar{q} g_{s} \sigma G q\right\rangle=m_{0}^{2}\langle\bar{q} q\rangle, m_{0}^{2}=$ $(0.8 \pm 0.1) \mathrm{GeV}^{2},\langle\bar{s} s\rangle=(0.8 \pm 0.1)\langle\bar{q} q\rangle,\left\langle\bar{s} g_{s} \sigma G s\right\rangle=$ $m_{0}^{2}\langle\bar{s} s\rangle,\left\langle\frac{\alpha_{s} G G}{\pi}\right\rangle=(0.33 \mathrm{GeV})^{4}$ at the energy scale $\mu=$ $1 \mathrm{GeV}[14-16,40]$, and choose the $\overline{M S}$ masses $m_{c}\left(m_{c}\right)=$ $(1.275 \pm 0.025) \mathrm{GeV}, m_{s}(\mu=2 \mathrm{GeV})=(0.095 \pm$ $0.005) \mathrm{GeV}$ from the Particle Data Group [41]. Moreover, we take into account the energy-scale dependence of the input parameters on the QCD side,

$$
\begin{aligned}
\langle\bar{q} q\rangle(\mu) & =\langle\bar{q} q\rangle(Q)\left[\frac{\alpha_{s}(Q)}{\alpha_{s}(\mu)}\right]^{\frac{4}{9}}, \\
\langle\bar{s} s\rangle(\mu) & =\langle\bar{s} s\rangle(Q)\left[\frac{\alpha_{s}(Q)}{\alpha_{s}(\mu)}\right]^{\frac{4}{9}}, \\
\left\langle\bar{q} g_{s} \sigma G q\right\rangle(\mu) & =\left\langle\bar{q} g_{s} \sigma G q\right\rangle(Q)\left[\frac{\alpha_{s}(Q)}{\alpha_{s}(\mu)}\right]^{\frac{2}{27}}, \\
\left\langle\bar{s} g_{s} \sigma G s\right\rangle(\mu)= & \left\langle\bar{s} g_{s} \sigma G s\right\rangle(Q)\left[\frac{\alpha_{s}(Q)}{\alpha_{s}(\mu)}\right]^{\frac{2}{27}}, \\
m_{c}(\mu)= & m_{c}\left(m_{c}\right)\left[\frac{\alpha_{s}(\mu)}{\alpha_{s}\left(m_{c}\right)}\right]^{\frac{12}{25}}, \\
m_{s}(\mu)= & m_{s}(2 \mathrm{GeV})\left[\frac{\alpha_{s}(\mu)}{\alpha_{s}(2 \mathrm{GeV})}\right]^{\frac{4}{9}}, \\
\alpha_{s}(\mu)= & \frac{1}{b_{0} t}\left[1-\frac{b_{1}}{b_{0}^{2}} \frac{\log t}{t}\right. \\
& \left.+\frac{b_{1}^{2}\left(\log { }^{2} t-\log t-1\right)+b_{0} b_{2}}{b_{0}^{4} t^{2}}\right],
\end{aligned}
$$


where $t=\log \frac{\mu^{2}}{\Lambda^{2}}, b_{0}=\frac{33-2 n_{f}}{12 \pi}, b_{1}=\frac{153-19 n_{f}}{24 \pi^{2}}, b_{2}=$ $\frac{2857-\frac{5033}{9} n_{f}+\frac{325}{27} n_{f}^{2}}{128 \pi^{3}}, \Lambda=213 \mathrm{MeV}, 296 \mathrm{MeV}$ and $339 \mathrm{MeV}$ for the flavors $n_{f}=5,4$ and 3, respectively [41], and evolve all the input parameters to the optimal energy scales $\mu$ to extract the masses of the doubly charmed tetraquark states $Z$ and $Y$.

In the article, we study the doubly charmed tetraquark states, the two charm quarks form an axialvector doubly charmed diquark state in color antitriplet, the axialvector doubly charmed diquark state serves as a static well potential and combines with an axialvector light antidiquark state in color triplet to form a compact tetraquark state. While in the hidden-charm tetraquark states, the charm quark $c$ serves as a static well potential and combines with the light quark $q$ to form a charmed diquark in color antitriplet, the charm antiquark $\bar{c}$ serves as another static well potential and combines with the light antiquark $\bar{q}^{\prime}$ to form a charmed antidiquark in color triplet, then the charmed diquark and charmed antidiquark combine together to form a hidden-charm tetraquark state. The quark structures of the doubly charmed tetraquark states and hidden-charm tetraquark states are quite different.

In Refs. [34-39], we studied the acceptable energy scales of the QCD spectral densities for the hidden-charm (hiddenbottom) tetraquark states and molecular states in the QCD sum rules in detail for the first time, and suggest an energyscale formula $\mu=\sqrt{M_{X / Y / Z}^{2}-\left(2 \mathbb{M}_{Q}\right)^{2}}$ to determine the optimal energy scales. The energy-scale formula also works well in studying the hidden-charm pentaquark states [42]. The updated values of the effective heavy quark masses are $\mathbb{M}_{c}=1.82 \mathrm{GeV}$ and $\mathbb{M}_{b}=5.17 \mathrm{GeV}[43,44]$. It is not necessary for the effective charm quark mass $\mathbb{M}_{c}$ in the doubly charmed tetraquark states to have the same value as the one in the hidden-charm tetraquark states. In calculations, we observe that if we choose a slightly different value $\mathbb{M}_{c}=1.84 \mathrm{GeV}$, the criteria of the QCD sum rules can be satisfied more easily. We obtain the energy-scale formula by setting the energy scale $\mu=V$, the virtuality $V$ (or bound energy not as robust) is defined by $V=\sqrt{M_{X / Y / Z}^{2}-\left(2 \mathbb{M}_{c}\right)^{2}}$ $[35,36]$. In this article, we take into account the $S U(3)$ breaking effect $m_{S}(\mu)$ by subtracting $m_{S}(\mu)$ from the virtuality $V$, $\mu_{k}=V_{k}=\sqrt{M_{X / Y / Z}^{2}-\left(2 \mathbb{M}_{c}\right)^{2}}-k m_{s}\left(\mu_{k}\right)$, where the numbers of the strange antiquark $\bar{s}$ in the doubly charmed tetraquark states are $k=0,1,2$.

In this article, we take the continuum threshold parameters as $\sqrt{s_{0}}=M_{Z / Y}+(0.4 \sim 0.7) \mathrm{GeV}$, and vary the parameters $\sqrt{s_{0}}$ to obtain the optimal Borel parameters $T^{2}$ to satisfy the following four criteria:

1. pole dominance on the phenomenological side;

2. convergence of the operator product expansion;

3. appearance of the Borel platforms;
4. satisfying the energy-scale formula.

The resulting Borel parameters or Borel windows $T^{2}$, continuum threshold parameters $s_{0}$, optimal energy scales of the QCD spectral densities, pole contributions of the ground states are shown explicitly in Table 1. From Table 1, we can see that the pole dominance can be well satisfied. The pole contributions PC are defined by

$\mathrm{PC}=\frac{\int_{4 m_{c}^{2}}^{s_{0}} \mathrm{~d} s \rho(s) \exp \left(-\frac{s}{T^{2}}\right)}{\int_{4 m_{c}^{2}}^{\infty} \mathrm{d} s \rho(s) \exp \left(-\frac{s}{T^{2}}\right)}$,

which decrease monotonously and quickly with increase of the Borel parameter $T^{2}$, as the continuum contributions are depressed by the factor $\exp \left(-\frac{s}{T^{2}}\right)$, large Borel parameter $T^{2}$ enhances the continuum contributions, the largest power of the QCD spectral densities $\rho(s) \propto s^{4}$, the convergent behaviors of the operator product expansion are not very good for the tetraquark states and molecular states. Furthermore, the pole contributions increase monotonously with increase of the threshold parameters $s_{0}$, the uncertainties of the threshold parameters $\delta \sqrt{s_{0}}= \pm 0.1 \mathrm{GeV}$ also lead to rather large variations of the pole contributions. So in the small Borel window $T_{\max }^{2}-T_{\min }^{2}=0.4 \mathrm{GeV}^{2}$ for the $J^{P}=(0 / 1 / 2)^{+}$ tetraquark states, the pole contributions vary in a rather large range, about (40-60)\%. Although the pole contributions have rather large uncertainties, $\mathrm{PC}=(50 \pm 10) \%$ for the $J^{P}=(0 / 1 / 2)^{+}$tetraquark states and PC $=(60 \pm 10) \%$ for the $J^{P}=1^{-}$tetraquark states, the pole dominance can be well satisfied, the predictions are reliable. On the other hand, if we choose larger energy scales $\mu$, the pole contributions are enhanced, the pole contributions are less sensitive to the Borel parameter $T^{2}$, however, we should determine the energy scales of the QCD spectral densities in a consistent way by using the energy-scale formula.

In Fig. 1, we plot the absolute contributions of the vacuum condensates $|D(n)|$ in the operator product expansion for the central values of the input parameters,

$$
D(n)=\frac{\int_{4 m_{c}^{2}}^{s_{0}} \mathrm{~d} s \rho_{n}(s) \exp \left(-\frac{s}{T^{2}}\right)}{\int_{4 m_{c}^{2}}^{s_{0}} \mathrm{~d} s \rho(s) \exp \left(-\frac{s}{T^{2}}\right)},
$$

where the $\rho_{n}(s)$ are the QCD spectral densities for the vacuum condensates of dimension $n$. From the figure, we can see that the dominant contributions come from the perturbative terms (or $D(0)$ ) for the $1^{-}$tetraquark states, the operator product expansion is well convergent, while in the case of the $0^{+}, 1^{+}$and $2^{+}$tetraquark states, the contributions of the vacuum condensates of dimension $n=6$ are very large, but the contributions of the vacuum condensates of dimensions 

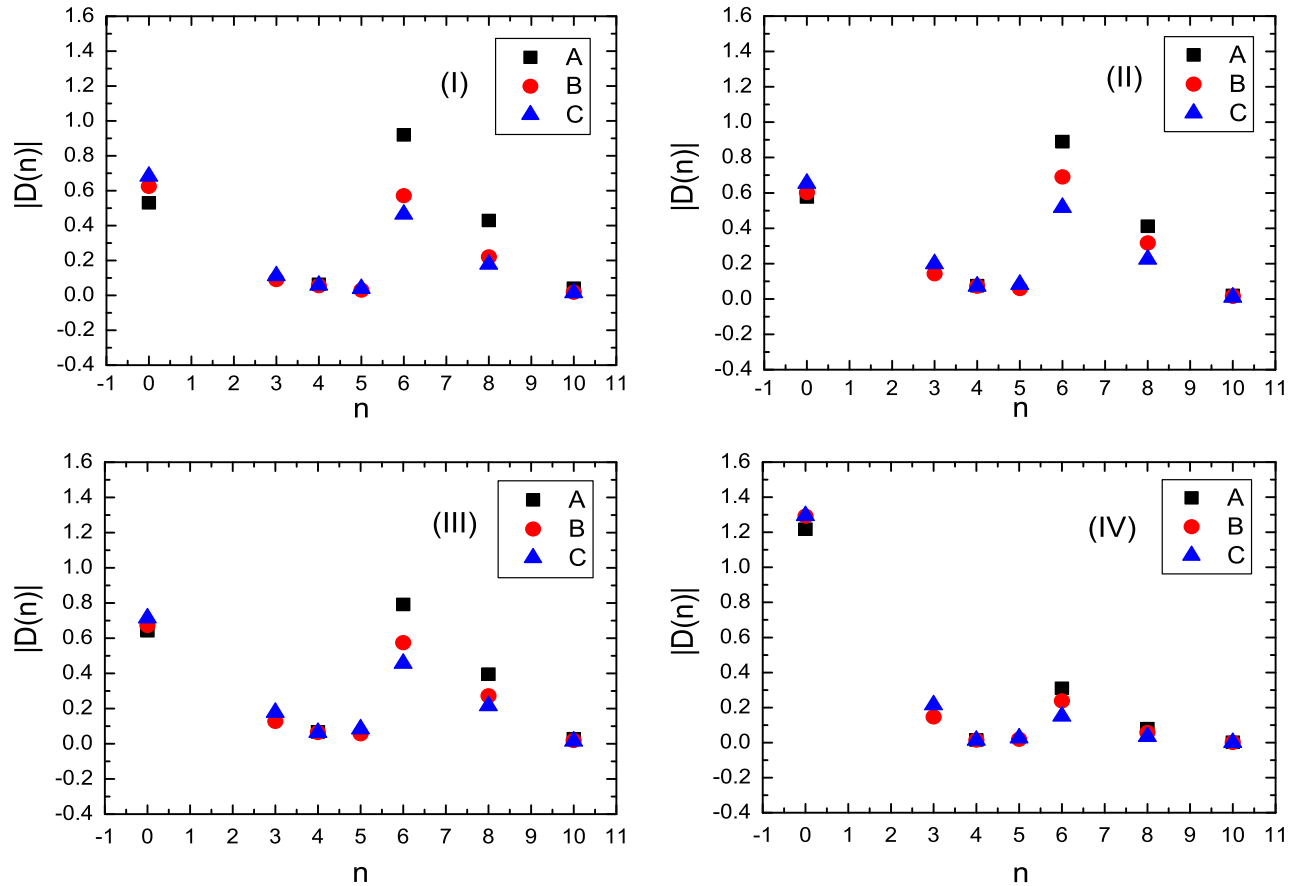

Fig. 1 The absolute contributions of the vacuum condensates of dimension $n$ for central values of the input parameters, where the (I)-(IV) denote the tetraquark states with $J^{P}=0^{+}, 1^{+}, 2^{+}$and $1^{-}$respectively, $A, B$ and $C$ denote the quark constituents $c c \bar{u} \bar{d}, c c \bar{u} \bar{s}$ and $c c \bar{s} \bar{s}$ respectively

Table 1 The Borel parameters (Borel windows), continuum threshold parameters, optimal energy scales, pole contributions, masses and pole residues for the doubly charmed tetraquark states

\begin{tabular}{lllllll}
\hline & $T^{2}\left(\mathrm{GeV}^{2}\right)$ & $\sqrt{s_{0}}(\mathrm{GeV})$ & $\mu(\mathrm{GeV})$ & Pole & $M(\mathrm{GeV})$ & $\lambda(\mathrm{GeV})$ \\
\hline $\operatorname{cc} \bar{u} \bar{d}\left(0^{+}\right)$ & $2.4-2.8$ & $4.40 \pm 0.10$ & 1.2 & $(38-63) \%$ & $3.87 \pm 0.09$ & $(3.90 \pm 0.63) \times 10^{-2}$ \\
$\operatorname{cc} \bar{u} \bar{s}\left(0^{+}\right)$ & $2.6-3.0$ & $4.50 \pm 0.10$ & 1.3 & $(38-62) \%$ & $3.94 \pm 0.10$ & $(4.92 \pm 0.89) \times 10^{-2}$ \\
$c c \bar{s} \bar{s}\left(0^{+}\right)$ & $2.6-3.0$ & $4.55 \pm 0.10$ & 1.3 & $(39-63) \%$ & $3.99 \pm 0.10$ & $(5.31 \pm 0.99) \times 10^{-2}$ \\
$\operatorname{cc} \bar{u} \bar{d}\left(1^{+}\right)$ & $2.6-3.0$ & $4.45 \pm 0.10$ & 1.3 & $(39-62) \%$ & $3.90 \pm 0.09$ & $(3.44 \pm 0.54) \times 10^{-2}$ \\
$c c \bar{u} \bar{s}\left(1^{+}\right)$ & $2.6-3.0$ & $4.50 \pm 0.10$ & 1.3 & $(40-64) \%$ & $3.96 \pm 0.08$ & $(3.78 \pm 0.59) \times 10^{-2}$ \\
$\operatorname{cc} \bar{s} \bar{s}\left(1^{+}\right)$ & $2.7-3.1$ & $4.55 \pm 0.10$ & 1.3 & $(39-62) \%$ & $4.02 \pm 0.09$ & $(4.11 \pm 0.68) \times 10^{-2}$ \\
$\operatorname{cc} \bar{u} \bar{d}\left(2^{+}\right)$ & $2.7-3.1$ & $4.50 \pm 0.10$ & 1.4 & $(39-62) \%$ & $3.95 \pm 0.09$ & $(5.67 \pm 0.90) \times 10^{-2}$ \\
$\operatorname{cc} \bar{u} \bar{s}\left(2^{+}\right)$ & $2.8-3.2$ & $4.55 \pm 0.10$ & 1.4 & $(38-60) \%$ & $4.01 \pm 0.09$ & $(6.27 \pm 1.02) \times 10^{-2}$ \\
$\operatorname{cc} \bar{s} \bar{s}\left(2^{+}\right)$ & $2.8-3.2$ & $4.60 \pm 0.10$ & 1.4 & $(39-61) \%$ & $4.06 \pm 0.09$ & $(6.78 \pm 1.12) \times 10^{-2}$ \\
$\operatorname{cc} \bar{u} \bar{d}\left(1^{-}\right)$ & $3.3-3.9$ & $5.20 \pm 0.10$ & 2.9 & $(50-73) \%$ & $4.66 \pm 0.10$ & $(1.31 \pm 0.17) \times 10^{-1}$ \\
$\operatorname{cc} \bar{u} \bar{s}\left(1^{-}\right)$ & $3.4-4.0$ & $5.25 \pm 0.10$ & 2.9 & $(49-71) \%$ & $4.73 \pm 0.11$ & $(1.40 \pm 0.19) \times 10^{-1}$ \\
$\operatorname{cc} \bar{s} \bar{s}\left(1^{-}\right)$ & $3.7-4.3$ & $5.30 \pm 0.10$ & 2.9 & $(49-72) \%$ & $4.78 \pm 0.11$ & $(1.48 \pm 0.19) \times 10^{-1}$ \\
\hline
\end{tabular}

$6,8,10$ have the hierarchy $|D(6)| \gg|D(8)| \gg|D(10)|$, the operator product expansion is also convergent.

We take into account all uncertainties of the input parameters, and obtain the values of the masses and pole residues of the doubly charmed tetraquark states $Z$ and $Y$, which are shown explicitly in Table 1 and Figs. 2, 3, 4, 5. In Figs. 2, 3, 4, 5 , we plot the masses and pole residues of the doubly charmed tetraquark states in much larger ranges than the Borel windows. From Figs. 2, 3, 4, 5, we can see that there appear platforms in the Borel windows shown in Table 1. Furthermore, from Table 1, we can see that the energy-scale formula $\mu_{k}=\sqrt{M_{X / Y / Z}^{2}-\left(2 \mathbb{M}_{c}\right)^{2}}-k m_{s}\left(\mu_{k}\right)$ with $k=0,1,2$ is also satisfied. Now the four criteria are all satisfied, and we expect to be able to make reliable predictions.

In Ref. [36], we tentatively assign the $Z_{c}(4020 / 4025)$ to be the $C \gamma_{\mu} \otimes \gamma_{\nu} C$ type hidden-charm axialvector tetraquark state, and we choose the current, 

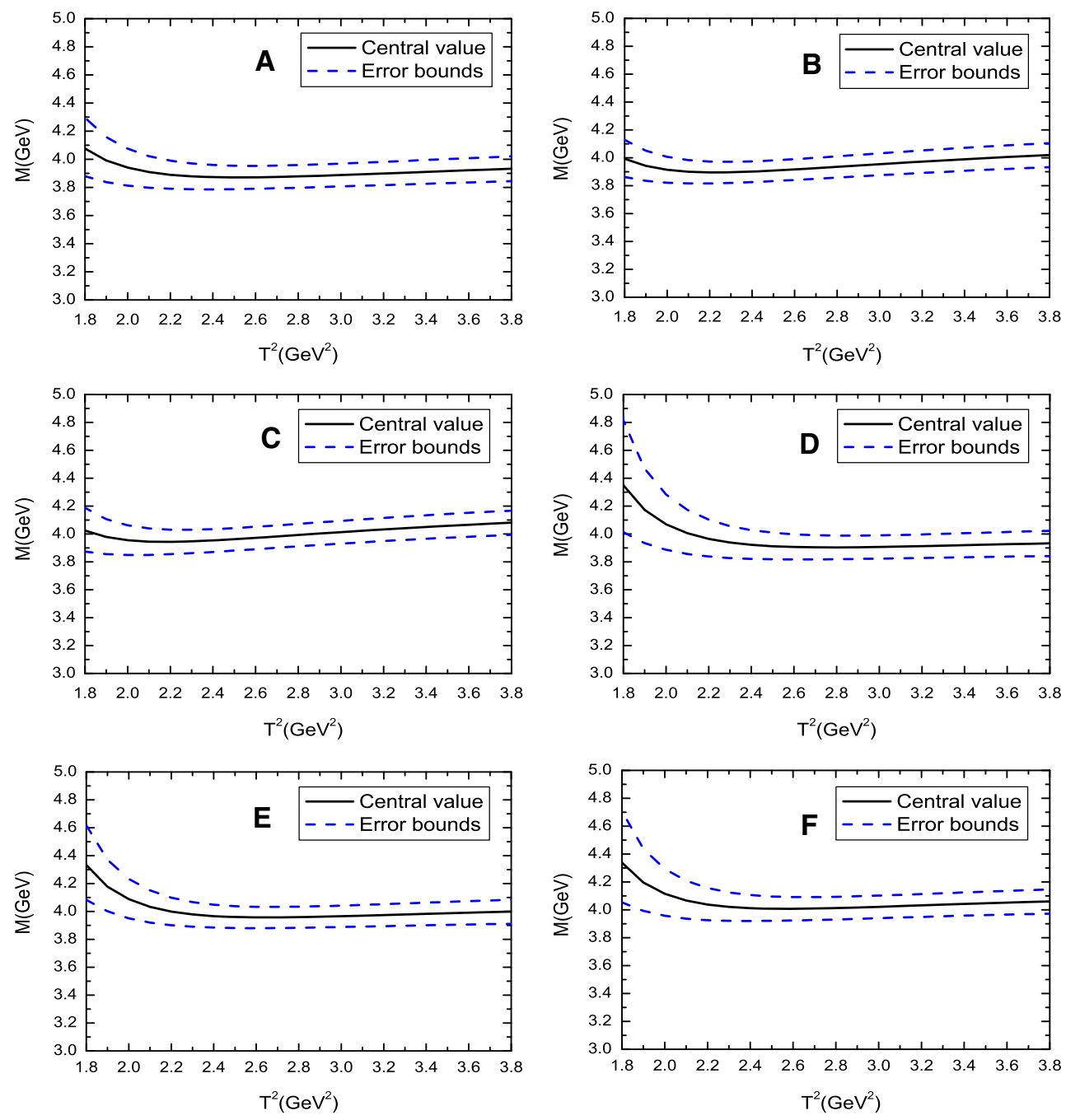

Fig. 2 The masses with variations of the Borel parameters, where a-f denote the tetraquark states $c c \bar{u} \bar{d}\left(0^{+}\right), c c \bar{u} \bar{s}\left(0^{+}\right), c c \bar{s} \bar{s}\left(0^{+}\right), c c \bar{u} \bar{d}\left(1^{+}\right)$, $c c \bar{u} \bar{s}\left(1^{+}\right)$and $\operatorname{cc} \bar{s} \bar{s}\left(1^{+}\right)$, respectively

$$
\begin{aligned}
J_{\mu \nu ; c \bar{c}}(x)= & \varepsilon^{i j k} \varepsilon^{i m n}\left\{u_{j}^{T}(x) C \gamma_{\mu} c_{k}(x) \bar{d}_{m}(x) \gamma_{\nu} C \bar{c}_{n}^{T}(x)\right. \\
& \left.-u_{j}^{T}(x) C \gamma_{\nu} c_{k}(x) \bar{d}_{m}(x) \gamma_{\mu} C \bar{c}_{n}^{T}(x)\right\},
\end{aligned}
$$

to study it with the QCD sum rules. In Ref. [33], we choose the axialvector current $J_{\mu ; c c}(x)$,

$$
J_{\mu ; c c}(x)=\varepsilon^{i j k} \varepsilon^{i m n} c_{i}^{T}(x) C \gamma_{\mu} c_{j}(x) \bar{u}_{m}(x) \gamma_{5} C \bar{d}_{n}^{T}(x),
$$

to study the $C \gamma_{\mu} \otimes \gamma_{5} C$ type doubly charmed tetraquark state with the QCD sum rules. In this article, we choose the axialvector current $J_{\mu \nu ; \bar{u}} \bar{d} ; 1(x)$,

$$
\begin{aligned}
& J_{\mu \nu ; \bar{u}} ; 1,1(x)= \\
& \varepsilon^{i j k} \varepsilon^{i m n}\left[c_{i}^{T}(x) C \gamma_{\mu} c_{j}(x) \bar{u}_{m}(x) \gamma_{\nu} C \bar{d}_{n}^{T}(x)\right. \\
&\left.-c_{i}^{T}(x) C \gamma_{\nu} c_{j}(x) \bar{u}_{m}(x) \gamma_{\mu} C \bar{d}_{n}^{T}(x)\right]
\end{aligned}
$$

to study the $C \gamma_{\mu} \otimes \gamma_{\nu} C$ type doubly charmed tetraquark state.
In Fig. 6, we plot the masses of the $C \gamma_{\mu} \otimes \gamma_{\nu} C$ type axialvector tetraquark state $c \bar{c} u \bar{d}, C \gamma_{\mu} \otimes \gamma_{5} C$ type axialvector tetraquark state $c c \bar{u} \bar{d}$ and $C \gamma_{\mu} \otimes \gamma_{\nu} C$ type axialvector tetraquark state $c c \bar{u} \bar{d}$ with variations of the Borel parameter $T^{2}$ for the energy scale $\mu=1.3 \mathrm{GeV}$ and continuum threshold parameter $\sqrt{s_{0}}=4.45 \mathrm{GeV}$. From the figure, we can see that the mass of the axialvector hidden-charm tetraquark state is $0.1 \mathrm{GeV}$ larger than the ones of the corresponding axialvector doubly charmed tetraquark states, while the $C \gamma_{\mu} \otimes \gamma_{5} C$ type and $C \gamma_{\mu} \otimes \gamma_{\nu} C$ type axialvector tetraquark states $c c \bar{u} \bar{d}$ have almost degenerate masses. In Ref. [36], we observe that the calculations based on the QCD sum rules support that $Z_{c}(4020 / 4025)$ can be assigned to the axialvector hiddencharm tetraquark state. So the $C \gamma_{\mu} \otimes \gamma_{5} C$ type and $C \gamma_{\mu} \otimes \gamma_{\nu} C$ type axialvector tetraquark states $c c \bar{u} \bar{d}$ have the masses about 3.9 GeV, the present predictions are reasonable. In Ref. [33] and present work, we observe that we can choose a universal effective $c$-quark mass $\mathbb{M}_{c}=1.84 \mathrm{GeV}$ to determine 

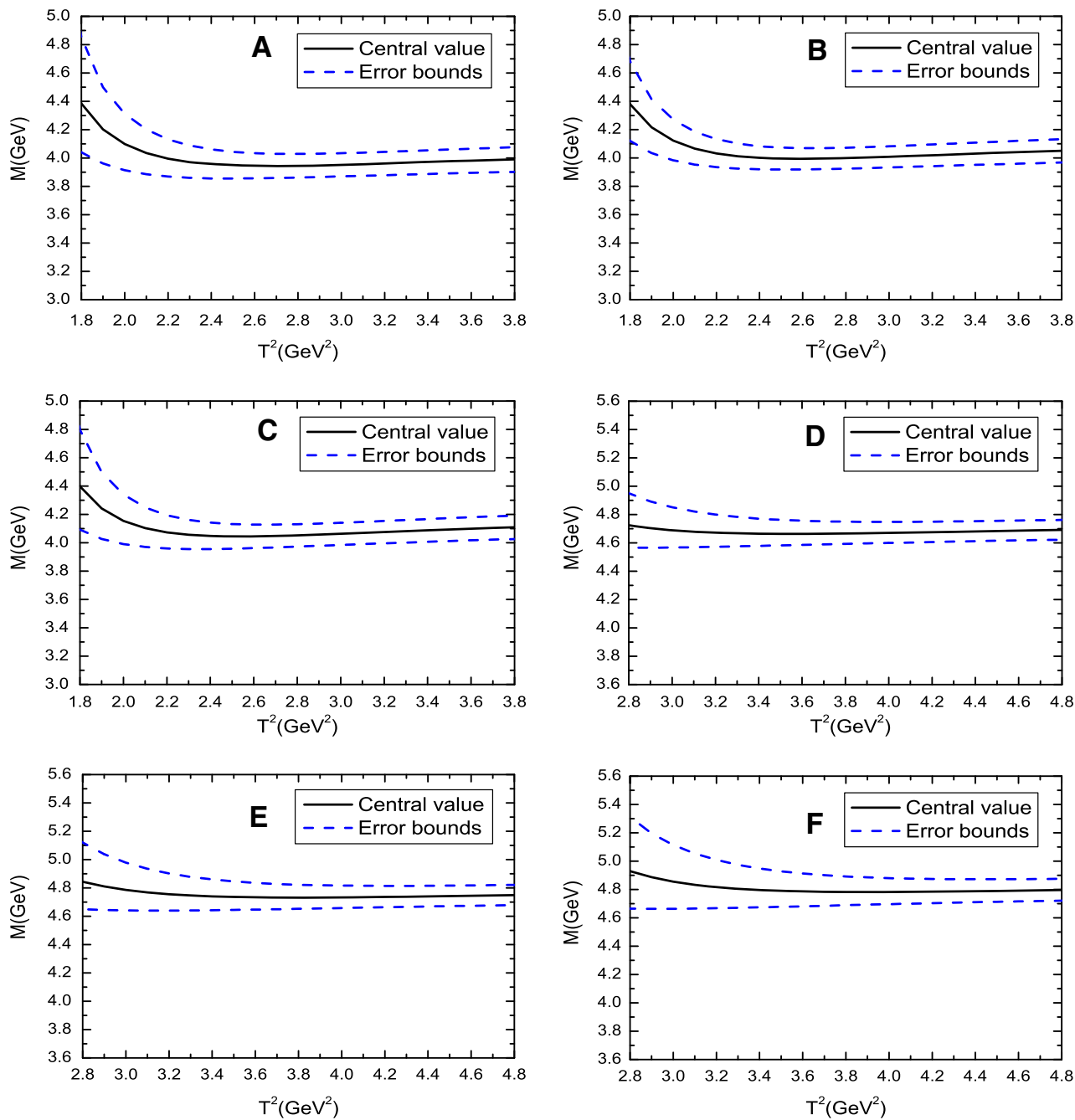

Fig. 3 The masses with variations of the Borel parameters, where a-f denote the tetraquark states $c c \bar{u} \bar{d}\left(2^{+}\right), c c \bar{u} \bar{s}\left(2^{+}\right), c c \bar{s} \bar{s}\left(2^{+}\right), c c \bar{u} \bar{d}\left(1^{-}\right)$, $c c \bar{u} \bar{s}\left(1^{-}\right)$and $c c \bar{s} \bar{s}\left(1^{-}\right)$, respectively

the energy scales of the QCD spectral densities in a consistent way, which leads to the energy scale $\mu=1.3 \mathrm{GeV}$ for the QCD spectral density of the $C \gamma_{\mu} \otimes \gamma_{5} C$ type tetraquark state $c c \bar{u} \bar{d}$. If we choose a slightly different energy scale $\mu=1.4 \mathrm{GeV}$ (which corresponds to a non-universal value $\mathbb{M}_{c}=1.82 \mathrm{GeV}$ ) and a slightly different threshold parameter, we can obtain the lowest mass $3.85 \pm 0.09 \mathrm{GeV}$, which is also shown in Table 1 in Ref. [33]. In this article, we prefer the universal effective $c$-quark mass $\mathbb{M}_{c}=1.84 \mathrm{GeV}$.

The centroids of the masses of the $C \gamma_{\mu} \otimes \gamma_{\nu} C$ type tetraquark states are

$$
\begin{aligned}
M_{C \gamma_{\mu} \otimes \gamma_{\nu} C}(c c \bar{u} \bar{d})= & \frac{M_{c c \bar{u} \bar{d} ; 0^{+}}+3 M_{c c \bar{u} \bar{d} ; 1^{+}}+5 M_{c c \bar{u} \bar{d} ; 2^{+}}}{9} \\
& =3.92 \mathrm{GeV}, \\
M_{C \gamma_{\mu} \otimes \gamma_{\nu} C}(c c \bar{u} \bar{s})= & \frac{M_{c c \bar{u} \bar{s} ; 0^{+}}+3 M_{c c \bar{u} \bar{s} ; 1^{+}}+5 M_{c c \bar{u} \bar{s} ; 2^{+}}}{9} \\
& =3.99 \mathrm{GeV},
\end{aligned}
$$

$$
\begin{aligned}
M_{C \gamma_{\mu} \otimes \gamma_{\nu} C}(c c \bar{s} \bar{s})= & \frac{M_{c c \bar{s} \bar{s} ; 0^{+}}+3 M_{c c \bar{s} \bar{s} ; 1^{+}}+5 M_{c c \bar{s} \bar{s} ; 2^{+}}}{9} \\
& =4.04 \mathrm{GeV},
\end{aligned}
$$

which are slightly larger than the centroids of the masses of the corresponding $C \gamma_{\mu} \otimes \gamma_{5} C$ type tetraquark states,

$$
\begin{aligned}
M_{C \gamma_{\mu} \otimes \gamma_{5} C}(c c \bar{u} \bar{d}) & =3.90 \mathrm{GeV}, \\
M_{C \gamma_{\mu} \otimes \gamma_{5} C}(c c \bar{u} \bar{s}) & =3.95 \mathrm{GeV},
\end{aligned}
$$

so the ground states are the $C \gamma_{\mu} \otimes \gamma_{5} C$ type tetraquark states, which is consistent with our naive expectation that the axialvector (anti)diquarks have larger masses than the corresponding scalar (anti)diquarks. The lowest centroids $M_{c c \bar{u} \bar{d} ; 0^{+}}=3.87 \mathrm{GeV}$ and $M_{c c \bar{u} \bar{s} ; 0^{+}}=3.94 \mathrm{GeV}$ originate from the spin splitting, in other words, the spin-spin interaction between the doubly heavy diquark and the light antidiquark. In fact, the predicted masses have uncertainties, the 

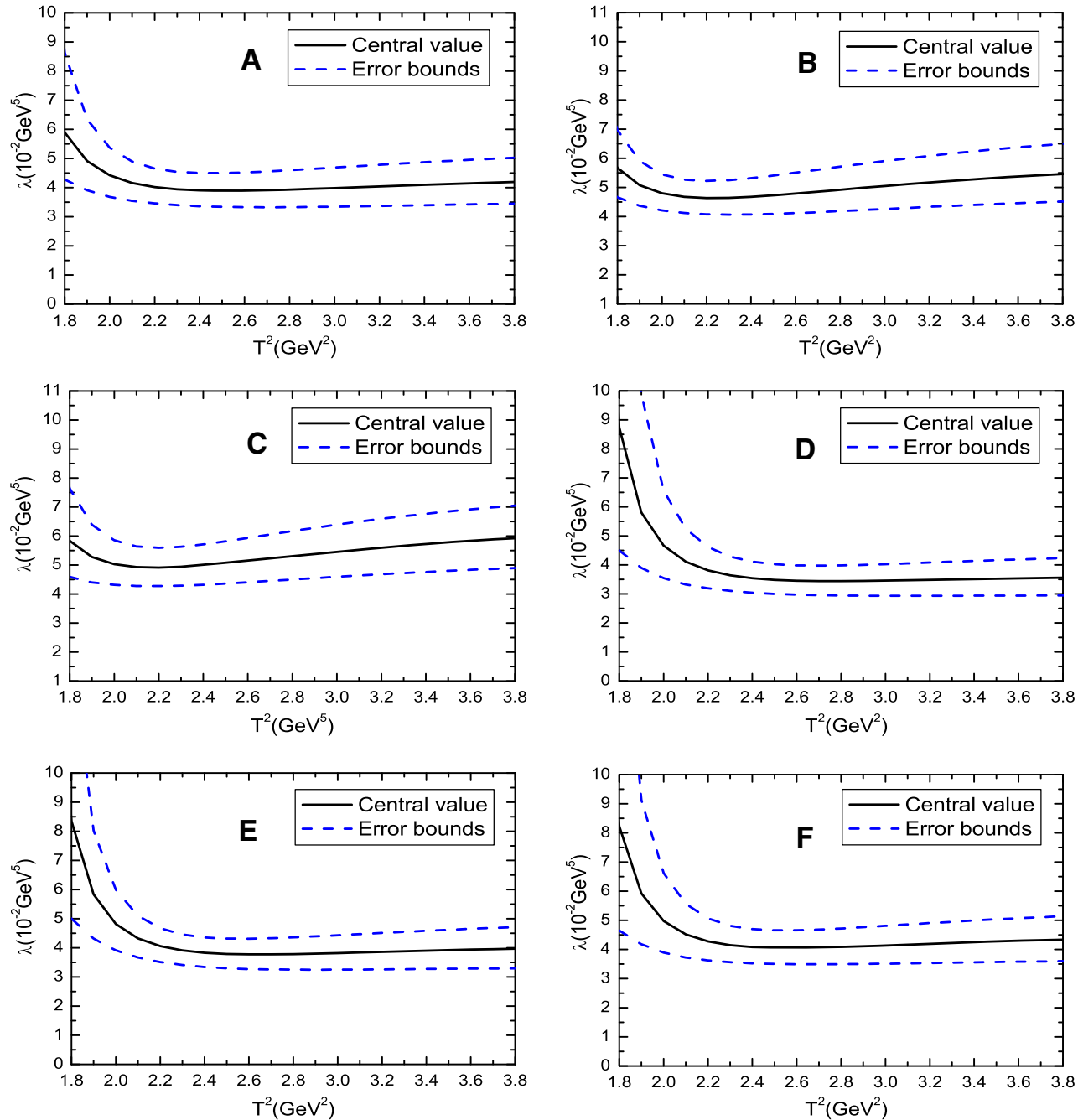

Fig. 4 The pole residues with variations of the Borel parameters, where a-f denote the tetraquark states $c c \bar{u} \bar{d}\left(0^{+}\right), c c \bar{u} \bar{s}\left(0^{+}\right), c c \bar{s} \bar{s}\left(0^{+}\right), c c \bar{u} \bar{d}\left(1^{+}\right)$, $\operatorname{cc} \bar{u} \bar{s}\left(1^{+}\right)$and $c c \bar{s} \bar{s}\left(1^{+}\right)$, respectively

centroids of the masses are not the super values, all values within uncertainties make sense.

In Ref. [29], Eichten and Quigg obtain the masses $M=$ $4146 \mathrm{MeV}, 4167 \mathrm{MeV}$ and $4210 \mathrm{MeV}$ for the $C \gamma_{\mu} \otimes \gamma_{\nu} C$ type axialvector tetraquark states $c c \bar{u} \bar{d}, c c \bar{u} \bar{s}$ and $c c \bar{s} \bar{s}$ respectively, which are about $0.20-0.25 \mathrm{GeV}$ larger than the central values of the present predictions. For the $C \gamma_{\mu} \otimes \gamma_{5} C$ type axialvector tetraquark state $c c \bar{u} \bar{d}$, Eichten and Quigg obtain the mass $M=3978 \mathrm{MeV}$ [29], which is $0.1 \mathrm{GeV}$ larger than the value $3882 \mathrm{MeV}$ obtained by Karliner and Rosner based on a simple potential quark model [23]. The present predictions are consistent with the value $3882 \mathrm{MeV}$ obtained by Karliner and Rosner.

The doubly charmed tetraquark states with $J^{P}=0^{+}$, $1^{+}$and $2^{+}$lie near the corresponding charmed-meson pair thresholds, the decays to the charmed-meson pairs are Okubo-Zweig-lizuka super-allowed,

$$
\begin{aligned}
Z_{c c \bar{u} \bar{d} ; 0^{+}} & \rightarrow D^{0} D^{+}, \\
Z_{c c \bar{u} \bar{s} ; 0^{+}} & \rightarrow D^{0} D_{s}^{+}, \\
Z_{c c \bar{s} \bar{s} ; 0^{+}} & \rightarrow D_{s}^{+} D_{s}^{+}, \\
Z_{c c \bar{u} \bar{d} ; 1^{+}} & \rightarrow D^{0} D^{*+}, D^{+} D^{* 0}, \\
Z_{c c \bar{u} \bar{s} ; 1^{+}} & \rightarrow D^{0} D_{s}^{*+}, D_{s}^{+} D^{* 0}, \\
Z_{c c \bar{s} \bar{s} ; 1^{+}} & \rightarrow D_{s}^{+} D_{s}^{*+}, \\
Z_{c c \bar{u} \bar{d} ; 2^{+}} & \rightarrow D^{0} D^{+}, D^{* 0} D^{*+}, \\
Z_{c c \bar{u} \bar{s} ; 2^{+}} & \rightarrow D^{0} D_{s}^{+}, \\
Z_{c c \bar{s} \bar{s} ; 2^{+}} & \rightarrow D_{s}^{+} D_{s}^{+},
\end{aligned}
$$

but the available phase spaces are very small, the decays are kinematically depressed, the doubly charmed tetraquark states with $J^{P}=0^{+}, 1^{+}$and $2^{+}$maybe have small widths. On the other hand, the doubly charmed tetraquark states with $J^{P}=1^{-}$lie above the corresponding charmed-meson 

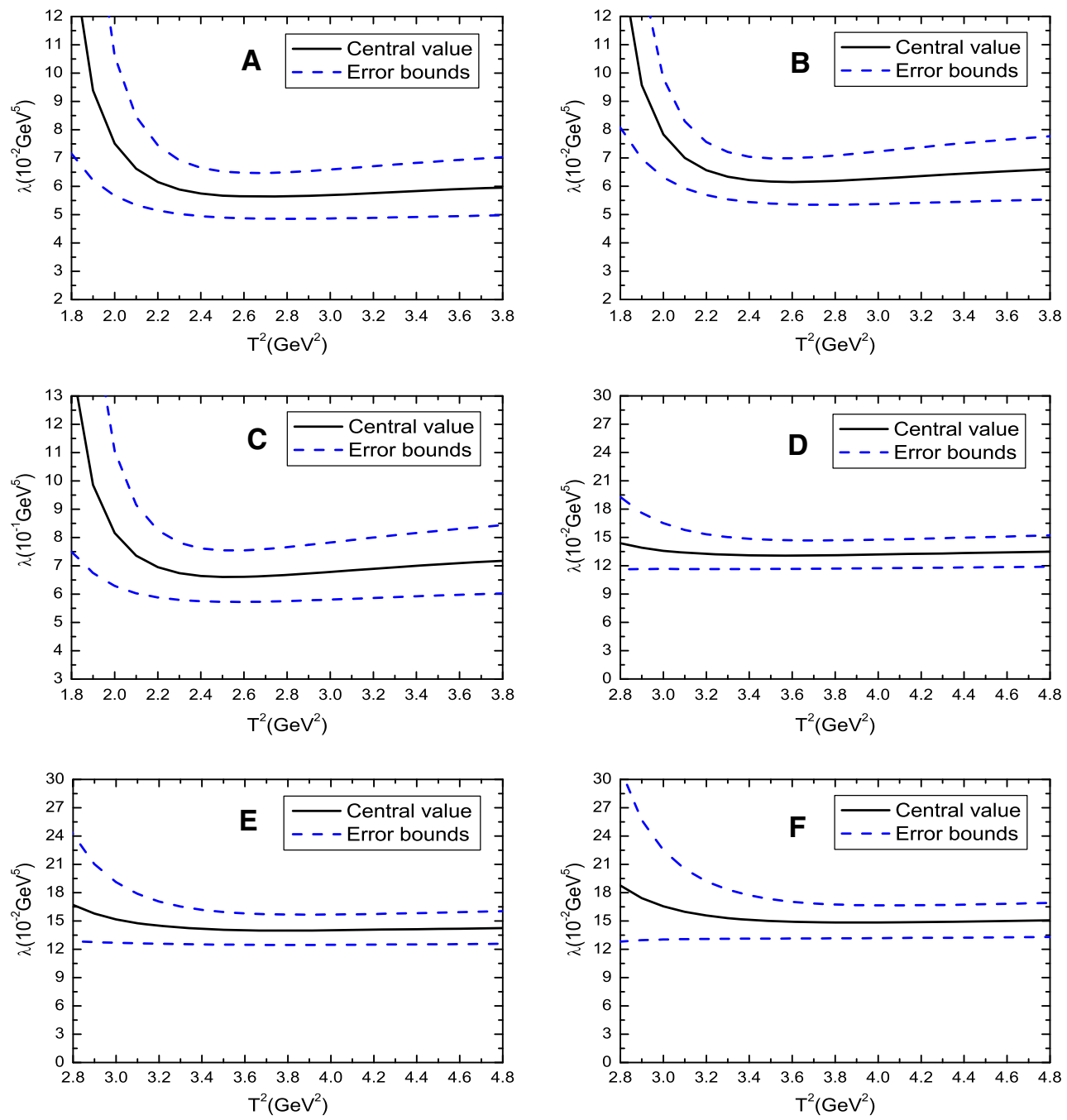

Fig. 5 The pole residues with variations of the Borel parameters, where a-f denote the tetraquark states $c c \bar{u} \bar{d}\left(2^{+}\right), c c \bar{u} \bar{s}\left(2^{+}\right), c c \bar{s} \bar{s}\left(2^{+}\right), c c \bar{u} \bar{d}\left(1^{-}\right)$, $c c \bar{u} \bar{s}\left(1^{-}\right)$and $c c \bar{s} \bar{s}\left(1^{-}\right)$, respectively

pair thresholds, the decays to the charmed-meson pairs are Okubo-Zweig-Iizuka super-allowed,

$$
\begin{aligned}
& Y_{c c \bar{u} \bar{d} ; 1^{-}} \rightarrow D^{0} D^{+}, D^{0} D^{*+}, D^{+} D^{* 0}, \\
& Y_{c c \bar{u} \bar{s} ; 1^{-}} \rightarrow D^{0} D_{s}^{+}, D^{0} D_{s}^{*+}, D_{s}^{+} D^{* 0}, \\
& Y_{c c \bar{s} \bar{s} ; 1^{-}} \rightarrow D_{s}^{+} D_{s}^{+}, D_{s}^{+} D_{s}^{*+},
\end{aligned}
$$

the available phase spaces are large, the decays are kinematically facilitated, the doubly charmed tetraquark states with the $J^{P}=1^{-}$should have large widths. We can search for the doubly charmed tetraquark states in those decay channels in the future.

\section{Conclusion}

In this article, we construct the axialvector-diquark-axialvector-antidiquark type currents to interpolate the scalar, axi- alvector, vector, tensor doubly charmed tetraquark states, and study them with the QCD sum rules in a systematic way. In calculations, we carry out the operator product expansion up to the vacuum condensates of dimension 10 consistently, then obtain the QCD spectral densities through dispersion relation, and extract the masses and pole residues in the Borel windows at the optimal energy scales of the QCD spectral densities, which are determined by the energy-scale formula with the refitted effective charm quark mass $\mathbb{M}_{c}$. In the Borel windows, the pole dominance is satisfied and the operator product expansion is well convergent, so we expect to make reliable predictions. We can search for those doubly charmed tetraquark states in the Okubo-Zweig-Iizuka superallowed strong decays to the charmed-meson pairs in the future. 


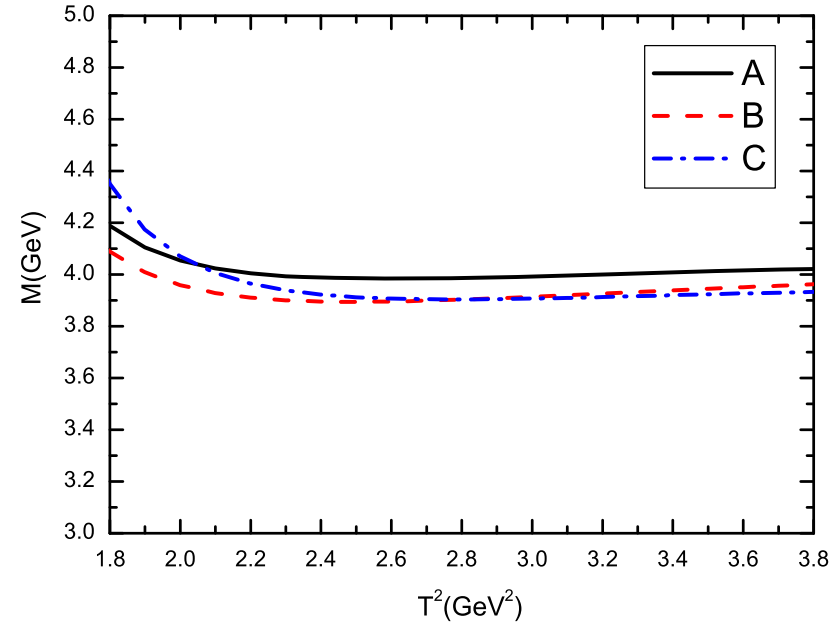

Fig. 6 The masses of the axialvector tetraquark states with variations of the Borel parameter $T^{2}$ for the energy scale $\mu=1.3 \mathrm{GeV}$ and continuum threshold parameter $\sqrt{s_{0}}=4.45 \mathrm{GeV}$, where $A, B$ and $C$ denote the $C \gamma_{\mu} \otimes \gamma_{\nu} C$ type tetraquark state $c \bar{c} u \bar{d}, C \gamma_{\mu} \otimes \gamma_{5} C$ type tetraquark state $c c \bar{u} \bar{d}$ and $C \gamma_{\mu} \otimes \gamma_{\nu} C$ type tetraquark state $c c \bar{u} \bar{d}$, respectively

Acknowledgements This work is supported by National Natural Science Foundation, Grant Number 11375063.

Open Access This article is distributed under the terms of the Creative Commons Attribution 4.0 International License (http://creativecomm ons.org/licenses/by/4.0/), which permits unrestricted use, distribution, and reproduction in any medium, provided you give appropriate credit to the original author(s) and the source, provide a link to the Creative Commons license, and indicate if changes were made.

Funded by SCOAP ${ }^{3}$.

\section{Appendix}

The explicit expressions of the QCD spectral densities $\rho_{\bar{u} \bar{d} ; 0}(s), \rho_{\bar{u} \bar{s} ; 0}(s), \rho_{\bar{s} \bar{s} ; 0}(s), \rho_{\bar{u} \bar{d} ; 1 ; A}(s), \rho_{\bar{u} \bar{s} ; 1 ; A}(s), \rho_{\bar{s} \bar{s} ; 1 ; A}(s)$, $\rho_{\bar{u} \bar{d} ; 1 ; V}(s), \quad \rho_{\bar{u} \bar{s} ; 1 ; V}(s), \quad \rho_{\bar{s} s ; 1 ; V}(s), \quad \rho_{\bar{u} \bar{d} ; 2}(s), \quad \rho_{\bar{u} \bar{s} ; 2}(s)$, $\rho_{\bar{s} \bar{s} ; 2}(s)$,

$$
\begin{aligned}
& \rho_{\bar{u} \bar{s} ; 0}(s)=\rho_{0 ; 0}(s)+\rho_{3 ; 0}(s)+\rho_{4 ; 0}(s) \\
& +\rho_{5 ; 0}(s)+\rho_{6 ; 0}(s)+\rho_{8 ; 0}(s)+\rho_{10 ; 0}(s), \\
& \rho_{\bar{u} \bar{s} ; 1 ; A}(s)=\rho_{0 ; 1 ; A}(s)+\rho_{3 ; 1 ; A}(s)+\rho_{4 ; 1 ; A}(s) \\
& +\rho_{5 ; 1 ; A}(s)+\rho_{6 ; 1 ; A}(s)+\rho_{8 ; 1 ; A}(s) \\
& +\rho_{10 ; 1 ; A}(s) \text {, } \\
& \rho_{\bar{u} \bar{s} ; 1 ; V}(s)=\rho_{0 ; 1 ; V}(s)+\rho_{3 ; 1 ; V}(s)+\rho_{4 ; 1 ; V}(s) \\
& +\rho_{5 ; 1 ; V}(s)+\rho_{6 ; 1 ; V}(s)+\rho_{8 ; 1 ; V}(s) \\
& +\rho_{10 ; 1 ; V}(s) \text {, } \\
& \begin{aligned}
\rho_{\bar{u} \bar{s} ; 2}(s)= & \rho_{0 ; 2}(s)+\rho_{3 ; 2}(s)+\rho_{4 ; 2}(s)+\rho_{5 ; 2}(s) \\
& +\rho_{6 ; 2}(s)+\rho_{8 ; 2}(s)+\rho_{10 ; 2}(s),
\end{aligned}
\end{aligned}
$$

$$
\begin{aligned}
\rho_{\bar{u} \bar{d} ; 1 ; V}(s) & =\left.\rho_{\bar{u} \bar{s} ; 1 ; V}(s)\right|_{m_{s} \rightarrow 0,\langle\bar{s} s\rangle \rightarrow\langle\bar{q} q\rangle,\left\langle\bar{s} g_{s} \sigma G s\right\rangle \rightarrow\left\langle\bar{q} g_{s} \sigma G q\right\rangle}, \\
\rho_{\bar{u} \bar{d} ; 2}(s) & =\left.\rho_{\bar{u} \bar{s} ; 2}(s)\right|_{m_{s} \rightarrow 0,\langle\bar{s} s\rangle \rightarrow\langle\bar{q} q\rangle,\left\langle\bar{s} g_{s} \sigma G s\right\rangle \rightarrow\left\langle\bar{q} g_{s} \sigma G q\right\rangle,}
\end{aligned}
$$

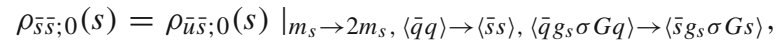

$\rho_{\bar{s} \bar{s} ; 1 ; A}(s)=\left.\rho_{\bar{u} \bar{s} ; 1 ; A}(s)\right|_{m_{s} \rightarrow 2 m_{s},\langle\bar{q} q\rangle \rightarrow\langle\bar{s} s\rangle,\left\langle\bar{q} g_{s} \sigma G q\right\rangle \rightarrow\left\langle\bar{s} g_{s} \sigma G s\right\rangle,}$,

$\rho_{\bar{s} \bar{s} ; 1 ; V}(s)=\left.\rho_{\bar{u} \bar{s} ; 1 ; V}(s)\right|_{m_{s} \rightarrow 2 m_{s},\langle\bar{q} q\rangle \rightarrow\langle\bar{s} s\rangle,\left\langle\bar{q} g_{s} \sigma G q\right\rangle \rightarrow\left\langle\bar{s} g_{s} \sigma G s\right\rangle,}$

$\rho_{\bar{s} \bar{s} ; 2}(s)=\left.\rho_{\bar{u} \bar{s} ; 2}(s)\right|_{m_{s} \rightarrow 2 m_{s},\langle\bar{q} q\rangle \rightarrow\langle\bar{s} s\rangle,\left\langle\bar{q} g_{s} \sigma G q\right\rangle \rightarrow\left\langle\bar{s} g_{s} \sigma G s\right\rangle}$,

$$
\begin{aligned}
\rho_{0 ; 0}(s)= & \frac{1}{64 \pi^{6}} \int_{y_{i}}^{y_{f}} \mathrm{~d} y \int_{z_{i}}^{1-y} \mathrm{~d} z y z(1-y-z)^{2}\left(s-\bar{m}_{c}^{2}\right)^{3} \\
& \times\left(3 s-\bar{m}_{c}^{2}\right) \\
& +\frac{m_{c}^{2}}{64 \pi^{6}} \int_{y_{i}}^{y_{f}} \mathrm{~d} y \int_{z_{i}}^{1-y} \mathrm{~d} z(1-y-z)^{2} \\
& \times\left(s-\bar{m}_{c}^{2}\right)^{3}, \\
\rho_{3 ; 0}(s)= & -\frac{m_{s}[\langle\bar{q} q\rangle-\langle\bar{s} s\rangle]}{4 \pi^{4}} \int_{y_{i}}^{y_{f}} \mathrm{~d} y \\
& \times \int_{z_{i}}^{1-y} \mathrm{~d} z y z\left(s-\bar{m}_{c}^{2}\right)\left(2 s-\bar{m}_{c}^{2}\right) \\
& -\frac{m_{s} m_{c}^{2}[4\langle\bar{q} q\rangle-\langle\bar{s} s\rangle]}{8 \pi^{4}} \int_{y_{i}}^{y_{f}} \mathrm{~d} y \\
& \times \int_{z_{i}}^{1-y} \mathrm{~d} z\left(s-\bar{m}_{c}^{2}\right),
\end{aligned}
$$$$
\rho_{4 ; 0}(s)=-\frac{m_{c}^{2}}{96 \pi^{4}}\left\langle\frac{\alpha_{s} G G}{\pi}\right\rangle \int_{y_{i}}^{y_{f}} \mathrm{~d} y \int_{z_{i}}^{1-y} \mathrm{~d} z
$$$$
\times\left(\frac{z}{y^{2}}+\frac{y}{z^{2}}\right)(1-y-z)^{2}\left(3 s-2 \bar{m}_{c}^{2}\right)
$$$$
-\frac{m_{c}^{4}}{192 \pi^{4}}\left\langle\frac{\alpha_{s} G G}{\pi}\right\rangle \int_{y_{i}}^{y_{f}} \mathrm{~d} y \int_{z_{i}}^{1-y} \mathrm{~d} z
$$$$
\times\left(\frac{1}{y^{3}}+\frac{1}{z^{3}}\right)(1-y-z)^{2}
$$$$
+\frac{m_{c}^{2}}{64 \pi^{4}}\left\langle\frac{\alpha_{s} G G}{\pi}\right\rangle \int_{y_{i}}^{y_{f}} \mathrm{~d} y \int_{z_{i}}^{1-y} \mathrm{~d} z
$$$$
\times\left[\left(\frac{1}{y^{2}}+\frac{1}{z^{2}}\right)(1-y-z)^{2}-1\right]\left(s-\bar{m}_{c}^{2}\right),
$$

$$
\rho_{5 ; 0}(s)=\frac{m_{s}\left[3\left\langle\bar{q} g_{s} \sigma G q\right\rangle-2\left\langle\bar{s} g_{s} \sigma G s\right\rangle\right]}{48 \pi^{4}}
$$




$$
\begin{array}{ll}
+\frac{m_{s} m_{c}^{2}\left[6\left\langle\bar{q} g_{s} \sigma G q\right\rangle-\left\langle\bar{s} g_{s} \sigma G s\right\rangle\right]}{48 \pi^{4}} \int_{y_{i}}^{y_{f}} \mathrm{~d} y & \times(1-y-z)^{3}\left(s-\bar{m}_{c}^{2}\right)^{2}\left(7 s-\bar{m}_{c}^{2}\right) \\
-\frac{m_{s}\left\langle\bar{q} g_{s} \sigma G q\right\rangle}{64 \pi^{4}} \int_{y_{i}}^{y_{f}} \mathrm{~d} y \int_{z_{i}}^{1-y} & +\frac{m_{c}^{2}}{192 \pi^{6}} \int_{y_{i}}^{y_{f}} \mathrm{~d} y \int_{z_{i}}^{1-y} \mathrm{~d} z(1-y-z)^{2} \\
\times \mathrm{d} z(y+z)\left(3 s-2 \bar{m}_{c}^{2}\right) & \times\left(s-\bar{m}_{c}^{2}\right)^{3}, \\
-\frac{m_{s} m_{c}^{2}\left\langle\bar{q} g_{s} \sigma G q\right\rangle}{32 \pi^{4}} \int_{y_{i}}^{y_{f}} \mathrm{~d} y \int_{z_{i}}^{1-y} \mathrm{~d} z\left(\frac{1}{y}+\frac{1}{z}\right), & \rho_{3 ; 1 ; A}(s)=\frac{m_{s}\langle\bar{s} s\rangle}{24 \pi^{4}} \int_{y_{i}}^{y_{f}} \mathrm{~d} y \int_{z_{i}}^{1-y} \mathrm{~d} z
\end{array}
$$

$-\frac{m_{s} m_{c}^{2}\left\langle\bar{q} g_{s} \sigma G q\right\rangle}{32 \pi^{4}} \int_{y_{i}}^{y_{f}} \mathrm{~d} y \int_{z_{i}}^{1-y} \mathrm{~d} z\left(\frac{1}{y}+\frac{1}{z}\right)$,

$$
\begin{aligned}
\rho_{6 ; 0}(s)= & \frac{\langle\bar{q} q\rangle\langle\bar{s} s\rangle}{\pi^{2}} \int_{y_{i}}^{y_{f}} \mathrm{~d} y y(1-y) s, \\
\rho_{8 ; 0}(s)= & -\frac{\langle\bar{s} s\rangle\left\langle\bar{q} g_{s} \sigma G q\right\rangle+\langle\bar{q} q\rangle\left\langle\bar{s} g_{s} \sigma G s\right\rangle}{4 \pi^{2}} \\
& \times \int_{y_{i}}^{y_{f}} \mathrm{~d} y y(1-y) \\
& \times\left[2+\left(2 s+\frac{s^{2}}{T^{2}}\right) \delta\left(s-\widetilde{m}_{c}^{2}\right)\right] \\
& +\frac{\langle\bar{s} s\rangle\left\langle\bar{q} g_{s} \sigma G q\right\rangle+\langle\bar{q} q\rangle\left\langle\bar{s} g_{s} \sigma G s\right\rangle}{48 \pi^{2}} \\
& \times \int_{y_{i}}^{y_{f}} \mathrm{~d} y\left[2+3 s \delta\left(s-\widetilde{m}_{c}^{2}\right)\right],
\end{aligned}
$$

$$
\begin{aligned}
\rho_{10 ; 0}(s)= & \frac{\left\langle\bar{q} g_{s} \sigma G q\right\rangle\left\langle\bar{s} g_{s} \sigma G s\right\rangle}{16 \pi^{2}} \\
& \times \int_{y_{i}}^{y_{f}} \mathrm{~d} y y(1-y) \\
& \times\left(2+\frac{2 s}{T^{2}}+\frac{s^{2}}{T^{4}}+\frac{s^{3}}{T^{6}}\right) \delta\left(s-\widetilde{m}_{c}^{2}\right) \\
& -\frac{\left\langle\bar{q} g_{s} \sigma G q\right\rangle\left\langle\bar{s} g_{s} \sigma G s\right\rangle}{96 \pi^{2}} \\
& \times \int_{y_{i}}^{y_{f}} \mathrm{~d} y\left(2+\frac{2 s}{T^{2}}+\frac{3 s^{2}}{T^{4}}\right) \delta\left(s-\widetilde{m}_{c}^{2}\right) \\
& +\frac{11\left\langle\bar{q} g_{s} \sigma G q\right\rangle\left\langle\bar{s} g_{s} \sigma G s\right\rangle}{768 \pi^{2}} \\
& \times \int_{y_{i}}^{y_{f}} \mathrm{~d} y\left(1+\frac{5 s}{T^{2}}\right) \delta\left(s-\widetilde{m}_{c}^{2}\right),
\end{aligned}
$$

$$
\begin{aligned}
\rho_{0 ; 1 ; A}(s)= & \frac{1}{384 \pi^{6}} \int_{y_{i}}^{y_{f}} \mathrm{~d} y \int_{z_{i}}^{1-y} \mathrm{~d} z y z(1-y-z)^{3} \\
& \times\left(s-\bar{m}_{c}^{2}\right)^{2}\left(21 s^{2}-14 s \bar{m}_{c}^{2}+\bar{m}_{c}^{4}\right) \\
& -\frac{1}{384 \pi^{6}} \int_{y_{i}}^{y_{f}} \mathrm{~d} y \int_{z_{i}}^{1-y} \mathrm{~d} z \\
& \times y z(1-y-z)^{2}\left(s-\bar{m}_{c}^{2}\right)^{3}\left(3 s-\bar{m}_{c}^{2}\right) \\
& +\frac{m_{c}^{2}}{576 \pi^{6}} \int_{y_{i}}^{y_{f}} \mathrm{~d} y \int_{z_{i}}^{1-y} \mathrm{~d} z
\end{aligned}
$$$$
\times y z(1-y-z)\left(25 s^{2}-24 s \bar{m}_{c}^{2}+3 \bar{m}_{c}^{4}\right)
$$$$
-\frac{m_{s}\langle\bar{q} q\rangle}{24 \pi^{4}} \int_{y_{i}}^{y_{f}} \mathrm{~d} y \int_{z_{i}}^{1-y} \mathrm{~d} z
$$$$
\times y z\left(s-\bar{m}_{c}^{2}\right)\left(5 s-\bar{m}_{c}^{2}\right)
$$$$
-\frac{m_{s}[2\langle\bar{q} q\rangle+\langle\bar{s} s\rangle]}{24 \pi^{4}} \int_{y_{i}}^{y_{f}} \mathrm{~d} y
$$$$
\times \int_{z_{i}}^{1-y} \mathrm{~d} z y z\left(s-\bar{m}_{c}^{2}\right)\left(2 s-\bar{m}_{c}^{2}\right)
$$$$
-\frac{m_{s} m_{c}^{2}[6\langle\bar{q} q\rangle-\langle\bar{s} s\rangle]}{24 \pi^{4}} \int_{y_{i}}^{y_{f}} \mathrm{~d} y
$$$$
\times \int_{z_{i}}^{1-y} \mathrm{~d} z\left(s-\bar{m}_{c}^{2}\right)
$$$$
+\frac{m_{s} m_{c}^{2}\langle\bar{s} s\rangle}{24 \pi^{4}} \int_{y_{i}}^{y_{f}} \mathrm{~d} y
$$$$
\times \int_{z_{i}}^{1-y} \mathrm{~d} z(1-y-z)\left(3 s-\bar{m}_{c}^{2}\right),
$$$$
\rho_{4 ; 1 ; A}(s)=-\frac{m_{c}^{2}}{288 \pi^{4}}\left\langle\frac{\alpha_{s} G G}{\pi}\right\rangle \int_{y_{i}}^{y_{f}} \mathrm{~d} y \int_{z_{i}}^{1-y} \mathrm{~d} z
$$$$
\times\left(\frac{z}{y^{2}}+\frac{y}{z^{2}}\right)(1-y-z)^{3}
$$$$
\times\left[4 s-\bar{m}_{c}^{2}+\frac{2 s^{2}}{3} \delta\left(s-\bar{m}_{c}^{2}\right)\right]
$$$$
+\frac{m_{c}^{2}}{576 \pi^{4}}\left\langle\frac{\alpha_{s} G G}{\pi}\right\rangle \int_{y_{i}}^{y_{f}} \mathrm{~d} y \int_{z_{i}}^{1-y} \mathrm{~d} z
$$$$
\times\left(\frac{z}{y^{2}}+\frac{y}{z^{2}}\right)(1-y-z)^{2}\left(3 s-2 \bar{m}_{c}^{2}\right)
$$$$
-\frac{m_{c}^{4}}{864 \pi^{4}}\left\langle\frac{\alpha_{s} G G}{\pi}\right\rangle \int_{y_{i}}^{y_{f}} \mathrm{~d} y \int_{z_{i}}^{1-y} \mathrm{~d} z
$$$$
\times\left(\frac{1}{y^{3}}+\frac{1}{z^{3}}\right)(1-y-z)^{3}\left[\frac{1}{2}+s \delta\left(s-\bar{m}_{c}^{2}\right)\right]
$$$$
+\frac{m_{c}^{2}}{576 \pi^{4}}\left\langle\frac{\alpha_{s} G G}{\pi}\right\rangle \int_{y_{i}}^{y_{f}} \mathrm{~d} y \int_{z_{i}}^{1-y} \mathrm{~d} z
$$ 


$$
\begin{aligned}
& \times(1-y-z)\left[1+\left(\frac{1}{y^{2}}+\frac{1}{z^{2}}\right)(1-y-z)^{2}\right] \\
& \times\left(3 s-\bar{m}_{c}^{2}\right) \\
& -\frac{m_{c}^{4}}{576 \pi^{4}}\left\langle\frac{\alpha_{s} G G}{\pi}\right\rangle \int_{y_{i}}^{y_{f}} \mathrm{~d} y \int_{z_{i}}^{1-y} \mathrm{~d} z \\
& \times\left(\frac{1}{y^{3}}+\frac{1}{z^{3}}\right)(1-y-z)^{2} \\
& +\frac{m_{c}^{2}}{576 \pi^{4}}\left\langle\frac{\alpha_{s} G G}{\pi}\right\rangle \int_{y_{i}}^{y_{f}} \mathrm{~d} y \int_{z_{i}}^{1-y} \mathrm{~d} z \\
& \times\left[\left(\frac{3}{y^{2}}+\frac{3}{z^{2}}\right)(1-y-z)^{2}-5\right]\left(s-\bar{m}_{c}^{2}\right) \\
& -\frac{1}{384 \pi^{4}}\left\langle\frac{\alpha_{s} G G}{\pi}\right\rangle \int_{y_{i}}^{y_{f}} \mathrm{~d} y \int_{z_{i}}^{1-y} \mathrm{~d} z \\
& \times(1-y-z)^{2}\left(s-\bar{m}_{c}^{2}\right)\left(2 s-\bar{m}_{c}^{2}\right) \\
& +\frac{1}{576 \pi^{4}}\left\langle\frac{\alpha_{s} G G}{\pi}\right\rangle \int_{y_{i}}^{y_{f}} \mathrm{~d} y \int_{z_{i}}^{1-y} \mathrm{~d} z \\
& \times y z(1-y-z)\left(25 s^{2}-24 s \bar{m}_{c}^{2}+3 \bar{m}_{c}^{4}\right) \\
& -\frac{1}{576 \pi^{4}}\left\langle\frac{\alpha_{s} G G}{\pi}\right\rangle \int_{y_{i}}^{y_{f}} \mathrm{~d} y \int_{z_{i}}^{1-y} \mathrm{~d} z \\
& \times y z\left(s-\bar{m}_{c}^{2}\right)\left(11 s-4 \bar{m}_{c}^{2}\right),
\end{aligned}
$$

$$
\begin{aligned}
\rho_{5 ; 1 ; A}(s)= & -\frac{m_{s}\left\langle\bar{s} g_{s} \sigma G s\right\rangle}{24 \pi^{4}} \int_{y_{i}}^{y_{f}} \mathrm{~d} y \int_{z_{i}}^{1-y} \mathrm{~d} z \\
& \times y z\left[4 s-\bar{m}_{c}^{2}+\frac{2 s^{2}}{3} \delta\left(s-\bar{m}_{c}^{2}\right)\right]
\end{aligned}
$$$$
+\frac{m_{s}\left\langle\bar{q} g_{s} \sigma G q\right\rangle}{48 \pi^{4}} \int_{y_{i}}^{y_{f}} \mathrm{~d} y y(1-y)\left(3 s-\tilde{m}_{c}^{2}\right)
$$$$
+\frac{m_{s}\left[3\left\langle\bar{q} g_{s} \sigma G q\right\rangle+\left\langle\bar{s} g_{s} \sigma G s\right\rangle\right]}{144 \pi^{4}}
$$$$
\times \int_{y_{i}}^{y_{f}} \mathrm{~d} y y(1-y)\left(3 s-2 \tilde{m}_{c}^{2}\right)
$$$$
-\frac{m_{s} m_{c}^{2}\left\langle\bar{s} g_{s} \sigma G s\right\rangle}{72 \pi^{4}} \int_{y_{i}}^{y_{f}} \mathrm{~d} y \int_{z_{i}}^{1-y} \mathrm{~d} z
$$$$
\times\left[\frac{1}{2}+s \delta\left(s-\bar{m}_{c}^{2}\right)\right]
$$$$
+\frac{m_{s} m_{c}^{2}\left[9\left\langle\bar{q} g_{s} \sigma G q\right\rangle-\left\langle\bar{s} g_{s} \sigma G s\right\rangle\right]}{144 \pi^{4}} \int_{y_{i}}^{y_{f}} \mathrm{~d} y
$$$$
-\frac{m_{s}\left\langle\bar{q} g_{s} \sigma G q\right\rangle}{192 \pi^{4}} \int_{y_{i}}^{y_{f}} \mathrm{~d} y \int_{z_{i}}^{1-y} \mathrm{~d} z
$$$$
\times(y+z)\left(2 s-\bar{m}_{c}^{2}\right)
$$

$$
\begin{aligned}
& -\frac{m_{s} m_{c}^{2}\left\langle\bar{q} g_{s} \sigma G q\right\rangle}{192 \pi^{4}} \int_{y_{i}}^{y_{f}} \mathrm{~d} y \\
& \times \int_{z_{i}}^{1-y} \mathrm{~d} z\left(\frac{1}{y}+\frac{1}{z}\right), \\
& \rho_{6 ; 1 ; A}(s)=\frac{2\langle\bar{q} q\rangle\langle\bar{s} s\rangle}{3 \pi^{2}} \int_{y_{i}}^{y_{f}} \mathrm{~d} y y(1-y) s, \\
& \rho_{8 ; 1 ; A}(s)=-\frac{\langle\bar{s} s\rangle\left\langle\bar{q} g_{s} \sigma G q\right\rangle+\langle\bar{q} q\rangle\left\langle\bar{s} g_{s} \sigma G s\right\rangle}{12 \pi^{2}} \\
& \times \int_{y_{i}}^{y_{f}} \mathrm{~d} y y(1-y) \\
& \times\left[3+\left(4 s+\frac{2 s^{2}}{T^{2}}\right) \delta\left(s-\tilde{m}_{c}^{2}\right)\right] \\
& +\frac{\langle\bar{s} s\rangle\left\langle\bar{q} g_{s} \sigma G q\right\rangle+\langle\bar{q} q\rangle\left\langle\bar{s} g_{s} \sigma G s\right\rangle}{144 \pi^{2}} \\
& \times \int_{y_{i}}^{y_{f}} \mathrm{~d} y\left[1+2 s \delta\left(s-\tilde{m}_{c}^{2}\right)\right], \\
& \rho_{10 ; 1 ; A}(s)=\frac{\left\langle\bar{q} g_{s} \sigma G q\right\rangle\left\langle\bar{s} g_{s} \sigma G s\right\rangle}{24 \pi^{2}} \\
& \times \int_{y_{i}}^{y_{f}} \mathrm{~d} y y(1-y) \\
& \times\left(\frac{s}{T^{2}}+\frac{s^{2}}{T^{4}}+\frac{s^{3}}{T^{6}}\right) \delta\left(s-\widetilde{m}_{c}^{2}\right) \\
& -\frac{\left\langle\bar{q} g_{s} \sigma G q\right\rangle\left\langle\bar{s} g_{s} \sigma G s\right\rangle}{288 \pi^{2}} \\
& \times \int_{y_{i}}^{y_{f}} \mathrm{~d} y\left(\frac{s}{T^{2}}+\frac{2 s^{2}}{T^{4}}\right) \delta\left(s-\widetilde{m}_{c}^{2}\right) \\
& -\frac{11\left\langle\bar{q} g_{s} \sigma G q\right\rangle\left\langle\bar{s} g_{s} \sigma G s\right\rangle}{768 \pi^{2}} \\
& \times \int_{y_{i}}^{y_{f}} \mathrm{~d} y \frac{s}{T^{2}} \delta\left(s-\tilde{m}_{c}^{2}\right), \\
& \rho_{0 ; 1 ; V}(s)=\frac{1}{384 \pi^{6}} \int_{y_{i}}^{y_{f}} \mathrm{~d} y \int_{z_{i}}^{1-y} \mathrm{~d} z \\
& \times y z(1-y-z)^{3}\left(s-\bar{m}_{c}^{2}\right)^{2} \\
& \times\left(21 s^{2}-14 s \bar{m}_{c}^{2}+\bar{m}_{c}^{4}\right) \\
& -\frac{1}{384 \pi^{6}} \int_{y_{i}}^{y_{f}} \mathrm{~d} y \int_{z_{i}}^{1-y} \mathrm{~d} z \\
& \times y z(1-y-z)^{2}\left(s-\bar{m}_{c}^{2}\right)^{3}\left(3 s-\bar{m}_{c}^{2}\right) \\
& +\frac{m_{c}^{2}}{576 \pi^{6}} \int_{y_{i}}^{y_{f}} \mathrm{~d} y \int_{z_{i}}^{1-y} \mathrm{~d} z \\
& \times(1-y-z)^{3}\left(s-\bar{m}_{c}^{2}\right)^{2}\left(7 s-\bar{m}_{c}^{2}\right) \\
& -\frac{m_{c}^{2}}{96 \pi^{6}} \int_{y_{i}}^{y_{f}} \mathrm{~d} y \int_{z_{i}}^{1-y} \mathrm{~d} z
\end{aligned}
$$




$$
\begin{aligned}
& \times(1-y-z)^{2}\left(s-\bar{m}_{c}^{2}\right)^{3}, \\
& \rho_{3 ; 1 ; V}(s)=\frac{m_{s}\langle\bar{s} s\rangle}{24 \pi^{4}} \int_{y_{i}}^{y_{f}} \mathrm{~d} y \int_{z_{i}}^{1-y} \mathrm{~d} z \\
& \times y z(1-y-z)\left(25 s^{2}-24 s \bar{m}_{c}^{2}+3 \bar{m}_{c}^{4}\right) \\
& -\frac{m_{s}\langle\bar{q} q\rangle}{24 \pi^{4}} \int_{y_{i}}^{y_{f}} \mathrm{~d} y \int_{z_{i}}^{1-y} \mathrm{~d} z \\
& \times y z\left(s-\bar{m}_{c}^{2}\right)\left(5 s-\bar{m}_{c}^{2}\right) \\
& +\frac{m_{s}[4\langle\bar{q} q\rangle-\langle\bar{s} s\rangle]}{24 \pi^{4}} \int_{y_{i}}^{y_{f}} \mathrm{~d} y \\
& \times \int_{z_{i}}^{1-y} \mathrm{~d} z y z\left(s-\bar{m}_{c}^{2}\right)\left(2 s-\bar{m}_{c}^{2}\right) \\
& +\frac{m_{s} m_{c}^{2}[4\langle\bar{q} q\rangle-\langle\bar{s} s\rangle]}{12 \pi^{4}} \int_{y_{i}}^{y_{f}} \mathrm{~d} y \\
& \times \int_{z_{i}}^{1-y} \mathrm{~d} z\left(s-\bar{m}_{c}^{2}\right) \\
& +\frac{m_{s} m_{c}^{2}\langle\bar{s} s\rangle}{24 \pi^{4}} \int_{y_{i}}^{y_{f}} \mathrm{~d} y \\
& \times \int_{z_{i}}^{1-y} \mathrm{~d} z(1-y-z)\left(3 s-\bar{m}_{c}^{2}\right) \text {, } \\
& \rho_{4 ; 1 ; V}(s)=-\frac{m_{c}^{2}}{288 \pi^{4}}\left\langle\frac{\alpha_{s} G G}{\pi}\right\rangle \int_{y_{i}}^{y_{f}} \mathrm{~d} y \int_{z_{i}}^{1-y} \mathrm{~d} z \\
& \times\left(\frac{z}{y^{2}}+\frac{y}{z^{2}}\right)(1-y-z)^{3} \\
& \times\left[4 s-\bar{m}_{c}^{2}+\frac{2 s^{2}}{3} \delta\left(s-\bar{m}_{c}^{2}\right)\right] \\
& +\frac{m_{c}^{2}}{576 \pi^{4}}\left\langle\frac{\alpha_{s} G G}{\pi}\right\rangle \int_{y_{i}}^{y_{f}} \mathrm{~d} y \int_{z_{i}}^{1-y} \mathrm{~d} z \\
& \times\left(\frac{z}{y^{2}}+\frac{y}{z^{2}}\right)(1-y-z)^{2}\left(3 s-2 \bar{m}_{c}^{2}\right) \\
& -\frac{m_{c}^{4}}{864 \pi^{4}}\left\langle\frac{\alpha_{s} G G}{\pi}\right\rangle \int_{y_{i}}^{y_{f}} \mathrm{~d} y \int_{z_{i}}^{1-y} \mathrm{~d} z \\
& \times\left(\frac{1}{y^{3}}+\frac{1}{z^{3}}\right)(1-y-z)^{3} \\
& \times\left[\frac{1}{2}+s \delta\left(s-\bar{m}_{c}^{2}\right)\right] \\
& +\frac{m_{c}^{2}}{576 \pi^{4}}\left\langle\frac{\alpha_{s} G G}{\pi}\right\rangle \int_{y_{i}}^{y_{f}} \mathrm{~d} y \int_{z_{i}}^{1-y} \mathrm{~d} z \\
& \times(1-y-z)\left[1+\left(\frac{1}{y^{2}}+\frac{1}{z^{2}}\right)(1-y-z)^{2}\right] \\
& \times\left(3 s-\bar{m}_{c}^{2}\right)
\end{aligned}
$$

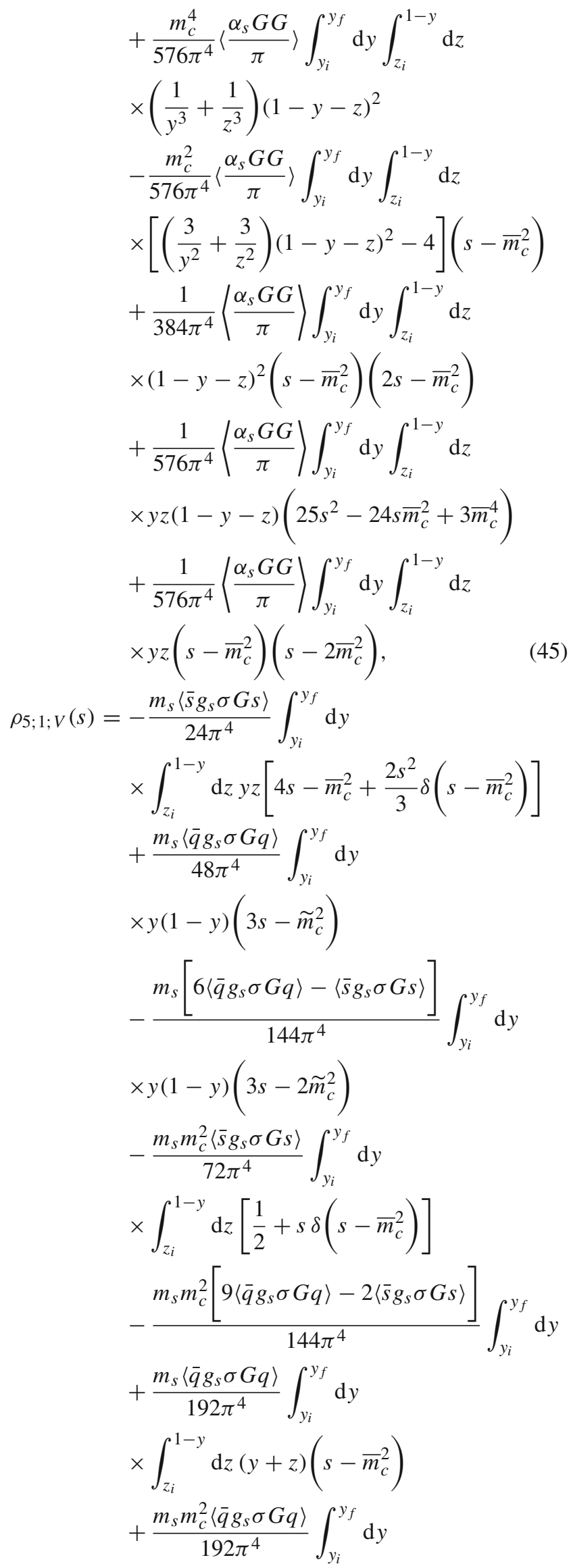


$\times \int_{z_{i}}^{1-y} \mathrm{~d} z\left(\frac{1}{y}+\frac{1}{z}\right)$

$\rho_{6 ; 1 ; V}(s)=-\frac{\langle\bar{q} q\rangle\langle\bar{s} s\rangle}{3 \pi^{2}} \int_{y_{i}}^{y_{f}} \mathrm{~d} y y(1-y) s$,

$\rho_{8 ; 1 ; V}(s)=\frac{\langle\bar{s} s\rangle\left\langle\bar{q} g_{s} \sigma G q\right\rangle+\langle\bar{q} q\rangle\left\langle\bar{s} g_{s} \sigma G s\right\rangle}{12 \pi^{2}}$

$\times \int_{y_{i}}^{y_{f}} \mathrm{~d} y y(1-y)$

$\times\left[3+\left(2 s+\frac{s^{2}}{T^{2}}\right) \delta\left(s-\tilde{m}_{c}^{2}\right)\right]$

$-\frac{\langle\bar{s} s\rangle\left\langle\bar{q} g_{s} \sigma G q\right\rangle+\langle\bar{q} q\rangle\left\langle\bar{s} g_{s} \sigma G s\right\rangle}{144 \pi^{2}}$

$\times \int_{y_{i}}^{y_{f}} \mathrm{~d} y\left[1+s \delta\left(s-\widetilde{m}_{c}^{2}\right)\right]$,

(48)

$\rho_{10 ; 1 ; V}(s)=-\frac{\left\langle\bar{q} g_{s} \sigma G q\right\rangle\left\langle\bar{s} g_{s} \sigma G s\right\rangle}{48 \pi^{2}}$

$\times \int_{y_{i}}^{y_{f}} \mathrm{~d} y y(1-y)$

$\times\left(6+\frac{4 s}{T^{2}}+\frac{s^{2}}{T^{4}}+\frac{s^{3}}{T^{6}}\right) \delta\left(s-\widetilde{m}_{c}^{2}\right)$

$+\frac{\left\langle\bar{q} g_{s} \sigma G q\right\rangle\left\langle\bar{s} g_{s} \sigma G s\right\rangle}{288 \pi^{2}}$

$\times \int_{y_{i}}^{y_{f}} \mathrm{~d} y\left(2+\frac{s}{T^{2}}+\frac{s^{2}}{T^{4}}\right) \delta\left(s-\tilde{m}_{c}^{2}\right)$

$+\frac{11\left\langle\bar{q} g_{s} \sigma G q\right\rangle\left\langle\bar{s} g_{s} \sigma G s\right\rangle}{2304 \pi^{2}}$

$\times \int_{y_{i}}^{y_{f}} \mathrm{~d} y\left(1+\frac{2 s}{T^{2}}\right) \delta\left(s-\tilde{m}_{c}^{2}\right)$,

(49)

$\rho_{0 ; 2}(s)=\frac{1}{960 \pi^{6}} \int_{y_{i}}^{y_{f}} \mathrm{~d} y \int_{z_{i}}^{1-y} \mathrm{~d} z y z(1-y-z)^{3}$

$\times\left(s-\bar{m}_{c}^{2}\right)^{2}\left(33 s^{2}-18 s \bar{m}_{c}^{2}+\bar{m}_{c}^{4}\right)$

$+\frac{1}{480 \pi^{6}} \int_{y_{i}}^{y_{f}} \mathrm{~d} y \int_{z_{i}}^{1-y} \mathrm{~d} z y z(1-y-z)^{2}$

$\times\left(s-\bar{m}_{c}^{2}\right)^{3}\left(9 s-2 \bar{m}_{c}^{2}\right)$

$+\frac{m_{c}^{2}}{288 \pi^{6}} \int_{y_{i}}^{y_{f}} \mathrm{~d} y \int_{z_{i}}^{1-y} \mathrm{~d} z(1-y-z)^{3}$

$\times\left(s-\bar{m}_{c}^{2}\right)^{2}\left(7 s-\bar{m}_{c}^{2}\right)$

$+\frac{m_{c}^{2}}{96 \pi^{6}} \int_{y_{i}}^{y_{f}} \mathrm{~d} y \int_{z_{i}}^{1-y} \mathrm{~d} z(1-y-z)^{2}$

$\times\left(s-\bar{m}_{c}^{2}\right)^{3}$,

$\rho_{3 ; 2}(s)=\frac{m_{s}\langle\bar{s} s\rangle}{60 \pi^{4}} \int_{y_{i}}^{y_{f}} \mathrm{~d} y \int_{z_{i}}^{1-y} \mathrm{~d} z$ $\times y z(1-y-z)\left(35 s^{2}-30 s \bar{m}_{c}^{2}+3 \bar{m}_{c}^{4}\right)$

$-\frac{m_{s}[5\langle\bar{q} q\rangle-\langle\bar{s} s\rangle]}{60 \pi^{4}} \int_{y_{i}}^{y_{f}} \mathrm{~d} y$

$\times \int_{z_{i}}^{1-y} \mathrm{~d} z y z\left(s-\bar{m}_{c}^{2}\right)\left(5 s-\bar{m}_{c}^{2}\right)$

$-\frac{m_{s}[10\langle\bar{q} q\rangle-3\langle\bar{s} s\rangle]}{60 \pi^{4}} \int_{y_{i}}^{y_{f}} \mathrm{~d} y$

$\times \int_{z_{i}}^{1-y} \mathrm{~d} z y z\left(s-\bar{m}_{c}^{2}\right)\left(2 s-\bar{m}_{c}^{2}\right)$

$-\frac{m_{s} m_{c}^{2}[6\langle\bar{q} q\rangle-\langle\bar{s} s\rangle]}{12 \pi^{4}} \int_{y_{i}}^{y_{f}} \mathrm{~d} y$

$\times \int_{z_{i}}^{1-y} \mathrm{~d} z\left(s-\bar{m}_{c}^{2}\right)$

$+\frac{m_{s} m_{c}^{2}\langle\bar{s} s\rangle}{12 \pi^{4}} \int_{y_{i}}^{y_{f}} \mathrm{~d} y \int_{z_{i}}^{1-y} \mathrm{~d} z$

$\times(1-y-z)\left(3 s-\bar{m}_{c}^{2}\right)$,

$\rho_{4 ; 2}(s)=-\frac{m_{c}^{2}}{720 \pi^{4}}\left\langle\frac{\alpha_{s} G G}{\pi}\right\rangle \int_{y_{i}}^{y_{f}} \mathrm{~d} y$

$\times \int_{z_{i}}^{1-y} \mathrm{~d} z\left(\frac{z}{y^{2}}+\frac{y}{z^{2}}\right)(1-y-z)^{3}$

$\times\left[5 s-\bar{m}_{c}^{2}+\frac{4 s^{2}}{3} \delta\left(s-\bar{m}_{c}^{2}\right)\right]$

$-\frac{m_{c}^{2}}{1440 \pi^{4}}\left\langle\frac{\alpha_{s} G G}{\pi}\right\rangle \int_{y_{i}}^{y_{f}} \mathrm{~d} y$

$\times \int_{z_{i}}^{1-y} \mathrm{~d} z\left(\frac{z}{y^{2}}+\frac{y}{z^{2}}\right)(1-y-z)^{2}$

$\times\left(15 s-8 \bar{m}_{c}^{2}\right)$

$-\frac{m_{c}^{4}}{432 \pi^{4}}\left\langle\frac{\alpha_{s} G G}{\pi}\right\rangle \int_{y_{i}}^{y_{f}} \mathrm{~d} y$

$\times \int_{z_{i}}^{1-y} \mathrm{~d} z\left(\frac{1}{y^{3}}+\frac{1}{z^{3}}\right)(1-y-z)^{3}$

$\times\left[\frac{1}{2}+s \delta\left(s-\bar{m}_{c}^{2}\right)\right]$

$+\frac{m_{c}^{2}}{288 \pi^{4}}\left\langle\frac{\alpha_{s} G G}{\pi}\right\rangle \int_{y_{i}}^{y_{f}} \mathrm{~d} y$

$\times \int_{z_{i}}^{1-y} \mathrm{~d} z(1-y-z)$

$\times\left[1+\left(\frac{1}{y^{2}}+\frac{1}{z^{2}}\right)(1-y-z)^{2}\right]$ 


$$
\begin{aligned}
& \times\left(3 s-\bar{m}_{c}^{2}\right) \\
& -\frac{m_{c}^{4}}{288 \pi^{4}}\left\langle\frac{\alpha_{s} G G}{\pi}\right\rangle \int_{y_{i}}^{y_{f}} \mathrm{~d} y \\
& \times \int_{z_{i}}^{1-y} \mathrm{~d} z\left(\frac{1}{y^{3}}+\frac{1}{z^{3}}\right)(1-y-z)^{2} \\
& +\frac{m_{c}^{2}}{288 \pi^{4}}\left\langle\frac{\alpha_{s} G G}{\pi}\right\rangle \int_{y_{i}}^{y_{f}} \mathrm{~d} y \\
& \times \int_{z_{i}}^{1-y} \mathrm{~d} z\left[\left(\frac{3}{y^{2}}+\frac{3}{z^{2}}\right)(1-y-z)^{2}-5\right] \\
& \times\left(s-\bar{m}_{c}^{2}\right) \\
& -\frac{1}{1440 \pi^{4}}\left\langle\frac{\alpha_{s} G G}{\pi}\right\rangle \int_{y_{i}}^{y_{f}} \mathrm{~d} y \\
& \times \int_{z_{i}}^{1-y} \mathrm{~d} z(1-y-z)^{3}\left(15 s^{2}-15 s \bar{m}_{c}^{2}+2 \bar{m}_{c}^{4}\right) \\
& -\frac{1}{960 \pi^{4}}\left\langle\frac{\alpha_{s} G G}{\pi}\right\rangle \int_{y_{i}}^{y_{f}} \mathrm{~d} y \\
& \times \int_{z_{i}}^{1-y} \mathrm{~d} z(1-y-z)^{2}\left(s-\bar{m}_{c}^{2}\right)\left(3 s-2 \bar{m}_{c}^{2}\right) \\
& +\frac{1}{1440 \pi^{4}}\left\langle\frac{\alpha_{s} G G}{\pi}\right\rangle \int_{y_{i}}^{y_{f}} \mathrm{~d} y \\
& \times \int_{z_{i}}^{1-y} \mathrm{~d} z y z(1-y-z) \\
& \times\left(35 s^{2}-30 s \bar{m}_{c}^{2}+3 \bar{m}_{c}^{4}\right) \\
& -\frac{1}{1440 \pi^{4}}\left\langle\frac{\alpha_{s} G G}{\pi}\right\rangle \int_{y_{i}}^{y_{f}} \mathrm{~d} y \\
& \times \int_{z_{i}}^{1-y} \mathrm{~d} z y z\left(s-\bar{m}_{c}^{2}\right)\left(34 s-11 \bar{m}_{c}^{2}\right) \text {, } \\
& \rho_{5 ; 2}(s)=-\frac{m_{s}\left\langle\bar{s} g_{s} \sigma G s\right\rangle}{60 \pi^{4}} \int_{y_{i}}^{y_{f}} \mathrm{~d} y \int_{z_{i}}^{1-y} \mathrm{~d} z \\
& \times y z\left[5 s-\bar{m}_{c}^{2}+\frac{4 s^{2}}{3} \delta\left(s-\bar{m}_{c}^{2}\right)\right] \\
& +\frac{m_{s}\left[15\left\langle\bar{q} g_{s} \sigma G q\right\rangle-2\left\langle\bar{s} g_{s} \sigma G s\right\rangle\right]}{360 \pi^{4}} \int_{y_{i}}^{y_{f}} \mathrm{~d} y \\
& \times y(1-y)\left(3 s-\tilde{m}_{c}^{2}\right) \\
& +\frac{m_{s}\left[5\left\langle\bar{q} g_{s} \sigma G q\right\rangle-\left\langle\bar{s} g_{s} \sigma G s\right\rangle\right]}{120 \pi^{4}} \int_{y_{i}}^{y_{f}} \mathrm{~d} y
\end{aligned}
$$$$
+\frac{m_{s} m_{c}^{2}\left[9\left\langle\bar{q} g_{s} \sigma G q\right\rangle-\left\langle\bar{s} g_{s} \sigma G s\right\rangle\right]}{72 \pi^{4}} \int_{y_{i}}^{y_{f}} \mathrm{~d} y
$$$$
-\frac{m_{s} m_{c}^{2}\left\langle\bar{s} g_{s} \sigma G s\right\rangle}{36 \pi^{4}} \int_{y_{i}}^{y_{f}} \mathrm{~d} y
$$$$
\times \int_{z_{i}}^{1-y} \mathrm{~d} z\left[\frac{1}{2}+s \delta\left(s-\bar{m}_{c}^{2}\right)\right]
$$$$
+\frac{m_{s}\left\langle\bar{q} g_{s} \sigma G q\right\rangle}{96 \pi^{4}} \int_{y_{i}}^{y_{f}} \mathrm{~d} y
$$$$
\times \int_{z_{i}}^{1-y} \mathrm{~d} z(y+z)\left(2 s-\bar{m}_{c}^{2}\right)
$$$$
+\frac{m_{s} m_{c}^{2}\left\langle\bar{q} g_{s} \sigma G q\right\rangle}{96 \pi^{4}} \int_{y_{i}}^{y_{f}} \mathrm{~d} y
$$$$
\times \int_{z_{i}}^{1-y} \mathrm{~d} z\left(\frac{1}{y}+\frac{1}{z}\right),
$$$$
\rho_{6 ; 2}(s)=\frac{4\langle\bar{q} q\rangle\langle\bar{s} s\rangle}{3 \pi^{2}} \int_{y_{i}}^{y_{f}} \mathrm{~d} y y(1-y) s,
$$$$
\rho_{8 ; 2}(s)=-\frac{\langle\bar{s} s\rangle\left\langle\bar{q} g_{s} \sigma G q\right\rangle+\langle\bar{q} q\rangle\left\langle\bar{s} g_{s} \sigma G s\right\rangle}{6 \pi^{2}}
$$$$
\times \int_{y_{i}}^{y_{f}} \mathrm{~d} y y(1-y)
$$$$
\times\left[3+\left(4 s+\frac{2 s^{2}}{T^{2}}\right) \delta\left(s-\tilde{m}_{c}^{2}\right)\right]
$$$$
-\frac{\langle\bar{s} s\rangle\left\langle\bar{q} g_{s} \sigma G q\right\rangle+\langle\bar{q} q\rangle\left\langle\bar{s} g_{s} \sigma G s\right\rangle}{72 \pi^{2}}
$$$$
\times \int_{y_{i}}^{y_{f}} \mathrm{~d} y\left[1+2 s \delta\left(s-\tilde{m}_{c}^{2}\right)\right],
$$$$
\rho_{10 ; 2}(s)=\frac{\left\langle\bar{q} g_{s} \sigma G q\right\rangle\left\langle\bar{s} g_{s} \sigma G s\right\rangle}{12 \pi^{2}}
$$$$
\times \int_{y_{i}}^{y_{f}} \mathrm{~d} y y(1-y)\left(\frac{s}{T^{2}}+\frac{s^{2}}{T^{4}}+\frac{s^{3}}{T^{6}}\right)
$$$$
\times \delta\left(s-\tilde{m}_{c}^{2}\right)
$$$$
+\frac{\left\langle\bar{q} g_{s} \sigma G q\right\rangle\left\langle\bar{s} g_{s} \sigma G s\right\rangle}{144 \pi^{2}}
$$$$
\times \int_{y_{i}}^{y_{f}} \mathrm{~d} y\left(\frac{s}{T^{2}}+\frac{2 s^{2}}{T^{4}}\right) \delta\left(s-\tilde{m}_{c}^{2}\right)
$$$$
+\frac{11\left\langle\bar{q} g_{s} \sigma G q\right\rangle\left\langle\bar{s} g_{s} \sigma G s\right\rangle}{1152 \pi^{2}}
$$$$
\times \int_{y_{i}}^{y_{f}} \mathrm{~d} y \frac{s}{T^{2}} \delta\left(s-\widetilde{m}_{c}^{2}\right),
$$

$y_{f}=\frac{1+\sqrt{1-4 m_{c}^{2} / s}}{2}, y_{i}=\frac{1-\sqrt{1-4 m_{c}^{2} / s}}{2}, z_{i}=\frac{y m_{c}^{2}}{y s-m_{c}^{2}}$, $\bar{m}_{c}^{2}=\frac{(y+z) m_{c}^{2}}{y z}, \widetilde{m}_{c}^{2}=\frac{m_{c}^{2}}{y(1-y)}, \int_{y_{i}}^{y_{f}} \mathrm{~d} y \rightarrow \int_{0}^{1} \mathrm{~d} y, \int_{z_{i}}^{1-y} \mathrm{~d} z \rightarrow$

$\int_{0}^{1-y} \mathrm{~d} z$ when the $\delta$ functions $\delta\left(s-\bar{m}_{c}^{2}\right)$ and $\delta\left(s-\tilde{m}_{c}^{2}\right)$ appear. 


\section{References}

1. R. Aaij et al., Phys. Rev. Lett. 119, 112001 (2017)

2. A. De Rujula, H. Georgi, S.L. Glashow, Phys. Rev. D 12, 147 (1975)

3. T. DeGrand, R.L. Jaffe, K. Johnson, J.E. Kiskis, Phys. Rev. D 12, $2060(1975)$

4. Z.G. Wang, Eur. Phys. J. C 71, 1524 (2011)

5. R.T. Kleiv, T.G. Steele, A. Zhang, Phys. Rev. D 87, 125018 (2013)

6. Z.G. Wang, Commun. Theor. Phys. 59, 451 (2013)

7. J.R. Zhang, M.Q. Huang, Phys. Rev. D 78, 094007 (2008)

8. Z.G. Wang, Eur. Phys. J. A 45, 267 (2010)

9. Z.G. Wang, Eur. Phys. J. C 68, 459 (2010)

10. S. Narison, R. Albuquerque, Phys. Lett. B 694, 217 (2011)

11. T.M. Aliev, K. Azizi, M. Savci, Nucl. Phys. A 895, 59 (2012)

12. T.M. Aliev, K. Azizi, M. Savci, J. Phys. G40, 065003 (2013)

13. H. X. Chen, Q. Mao, W. Chen, X. Liu, S. L. Zhu, arXiv:1707.01779

14. M.A. Shifman, A.I. Vainshtein, V.I. Zakharov, Nucl. Phys. B 147, 385 (1979)

15. M.A. Shifman, A.I. Vainshtein, V.I. Zakharov, Nucl. Phys. B 147, 448 (1979)

16. L.J. Reinders, H. Rubinstein, S. Yazaki, Phys. Rept. 127, 1 (1985)

17. S. Pepin, F. Stancu, M. Genovese, J.M. Richard, Phys. Lett. B 393, 119 (1997)

18. D. Ebert, R.N. Faustov, V.O. Galkin, W. Lucha, Phys. Rev. D 76, 114015 (2007)

19. Y. Yang, C. Deng, J. Ping, T. Goldman, Phys. Rev. D 80, 114023 (2009)

20. J. Vijande, A. Valcarce, N. Barnea, Phys. Rev. D 79, 074010 (2009)

21. T.F. Carames, A. Valcarce, J. Vijande, Phys. Lett. B 699, 291 (2011)
22. S. Q. Luo, K. Chen, X. Liu, Y.R. Liu, S. L. Zhu, arXiv:1707.01180

23. M. Karliner, J.L. Rosner, arXiv:1707.07666

24. F.S. Navarra, M. Nielsen, S.H. Lee, Phys. Lett. B 649, 166 (2007)

25. Z.G. Wang, Y.M. Xu, H.J. Wang, Commun. Theor. Phys. 55, 1049 (2011)

26. M.L. Du, W. Chen, X.L. Chen, S.L. Zhu, Phys. Rev. D 87, 014003 (2013)

27. A.V. Manohar, M.B. Wise, Nucl. Phys. B 399, 17 (1993)

28. M. Karliner, S. Nussinov, JHEP 1307, 153 (2013)

29. E. J. Eichten, C. Quigg, arXiv: 1707.09575

30. P. Bicudo, K. Cichy, A. Peters, B. Wagenbach, M. Wagner, Phys. Rev. D 92, 014507 (2015)

31. P. Bicudo, J. Scheunert, M. Wagner, Phys. Rev. D 95, 034502 (2017)

32. A. Francis, R.J. Hudspith, R. Lewis, K. Maltman, Phys. Rev. Lett. 118, 142001 (2017)

33. Z.G. Wang, arXiv: 1708.04545

34. Z.G. Wang, T. Huang, Phys. Rev. D 89, 054019 (2014)

35. Z.G. Wang, Eur. Phys. J. C 74, 2874 (2014)

36. Z.G. Wang, Commun. Theor. Phys. 63, 466 (2015)

37. Z.G. Wang, T. Huang, Nucl. Phys. A 930, 63 (2014)

38. Z.G. Wang, T. Huang, Eur. Phys. J. C 74, 2891 (2014)

39. Z.G. Wang, Eur. Phys. J. C 74, 2963 (2014)

40. P. Colangelo, A. Khodjamirian, arXiv:hep-ph/0010175

41. C. Patrignani et al., Chin. Phys. C 40, 100001 (2016)

42. Z.G. Wang, Eur. Phys. J. C 76, 70 (2016)

43. Z.G. Wang, Eur. Phys. J. C 76, 387 (2016)

44. Z.G. Wang, Commun. Theor. Phys. 66, 335 (2016) 